\title{
A TWO-SIDED LAPLACE INVERSION ALGORITHM WITH COMPUTABLE ERROR BOUNDS AND ITS APPLICATIONS IN FINANCIAL ENGINEERING
}

\author{
NING CAI,* The Hong Kong University of Science and Technology \\ S. G. KOU,** National University of Singapore and Columbia University \\ ZONGJIAN LIU, ${ }^{* * *}$ Columbia University
}

\begin{abstract}
Transform-based algorithms have wide applications in applied probability, but rarely provide computable error bounds to guarantee the accuracy. We propose an inversion algorithm for two-sided Laplace transforms with computable error bounds. The algorithm involves a discretization parameter $C$ and a truncation parameter $N$. By choosing $C$ and $N$ using the error bounds, the algorithm can achieve any desired accuracy. In many cases, the bounds decay exponentially, leading to fast computation. Therefore, the algorithm is especially suitable to provide benchmarks. Examples from financial engineering, including valuation of cumulative distribution functions of asset returns and pricing of European and exotic options, show that our algorithm is fast and easy to implement.
\end{abstract}

Keywords: Laplace inversion; two-sided Laplace transform; option pricing; discretization error; truncation error

2010 Mathematics Subject Classification: Primary 91G20

Secondary 44A10

\section{Introduction}

Transform-based numerical methods have been widely applied in financial engineering, including the one-sided Laplace transform in [1], the Fourier transform in [6], the fast Gaussian transform in [2], the Hilbert transform in [9] and [10], and the sinc expansion in [8]. These transform-based methods are designed for different purposes. For example, if the objective is to compute the prices of discrete barrier and lookback options when the return distribution is Gaussian or a mixture of independent Gaussian random variables, then the fast Gaussian transform algorithm of Broadie and Yamamoto [2] is preferable, since it is extremely fast; the Hilbert transform method of Feng and Linetsky [9] and [10] is especially useful to compute expectations that involve indicator functions under general Lévy processes.

In many financial applications, two-sided (rather than one-sided) Laplace transforms on the whole real line are available. In this paper we propose an inversion algorithm with computable error bounds for two-sided Laplace transforms. Although most transform-based methods can lead to fast computation, it is rare to have computable error bounds to guarantee accuracy.

Received 25 September 2012; revision received 25 July 2013.

* Postal address: Department of Industrial Engineering and Logistics Management, The Hong Kong University of Science and Technology, Room 5551, Clear Water Bay, Kowloon, Hong Kong. Email address: ningcai@ust.hk

** Postal address: Risk Management Institute and Department of Mathematics, National University of Singapore, I3 Building \#04-03, 21 Heng Mui Keng Terrace, Singapore 119613.

*** Postal address: Columbia University, 313A S. W. Mudd Building, 500 W. 120th Street, New York, NY 10027, USA. 
The contribution of this paper is threefold. (i) We derive a two-sided Laplace inversion formula that involves a discretization parameter $C$ and a truncation parameter $N$; see Theorem 3.1. (ii) The bounds of both the discretization and truncation errors are computable (see Theorem 4.1 and Theorem 5.1), so that, by choosing $C$ and $N$ using the error bounds, the algorithm can achieve any desired accuracy. Therefore, the algorithm is especially suitable to provide benchmarks. (iii) In many cases, the error bounds decay exponentially, leading to fast computation.

Petrella [14] proposed a two-sided Laplace inversion algorithm with a scaling factor (similar to our discretization parameter) without rigorous justification. We show that, unfortunately, Petrella's method imposes a constraint on the scaling factor. As a result, Petrella's method may lead to large errors; see Example 4.2 in Section 4.3. In addition, Petrella [14] did not analyze truncation errors.

Applications of the Fourier inversion method in finance date back to Carr and Madan [6]; see also [12]. As pointed out by Carr and Madan [7], the Fourier inversion method breaks down for deep out-of-the-money options and even generates negative values. Numerical examples demonstrate that our two-sided Laplace inversion algorithm can produce accurate option prices even when the Fourier inversion method fails; see, e.g. Table 5.

Feng and Lin [8] developed an elegant sinc expansion for the analytic characteristic function $(\mathrm{CF})$ of the asset return (or general continuous random variables) and then used this sinc expansion to approximate its probability density function (PDF), its cumulative distribution function $(\mathrm{CDF})$, and the related European option price by expressing these quantities as certain integrals of the CF. Numerical experiments indicate that this method is very accurate, efficient, and robust. However, their approximation formulae for different quantities may vary greatly because they depend on how these quantities are expressed as integrals of the CF. For example, they approximate the PDF by representing it as the inverse Fourier transform of the CF, while they approximate the CDF and the European option price by expressing them in terms of the Hilbert transform of the CF. By contrast, our two-sided Laplace inversion formula remains the same for different quantities and, hence, can be implemented simply in the same way.

The remainder of the paper is organized as follows. In Section 2 we present basic definitions. In Section 3 we provide a two-sided Laplace inversion formula. In Section 4 and Section 5 we study discretization and truncation errors, respectively. Applications in financial engineering are discussed in Sections 6 and 7, and numerical examples are given in Section 8. All the proofs are deferred to the appendices or the electronic companion [4].

\section{Preliminary results}

\subsection{Two-sided Laplace transforms and the region of absolute convergence}

Consider a function $f(t)$ defined for $t \in(-\infty,+\infty)$. Its two-sided or bilateral Laplace transform is the function $L_{f}(s): \mathbb{C} \mapsto \mathbb{C}$, defined as

$$
L_{f}(s):=\int_{-\infty}^{\infty} \mathrm{e}^{-s t} f(t) \mathrm{d} t
$$

for a complex number $s=\sigma+\mathrm{i} \omega \equiv \operatorname{Re}(s)+\mathrm{i} \operatorname{Im}(s)$. The region of absolute convergence (ROAC) is the set of $s$ such that

$$
\int_{-\infty}^{\infty} \mathrm{e}^{-\sigma t}|f(t)| \mathrm{d} t<+\infty .
$$


Clearly, if $s \in$ ROAC then the two-sided Laplace transform in (2.1) is well defined. Since the ROAC does not depend on $\operatorname{Im}(s)$, we may simply express the ROAC by the range of $\operatorname{Re}(s)$. If we define

$$
\begin{gathered}
\sigma_{1}:=\inf \left\{\sigma \in \mathbb{R}: \int_{-\infty}^{\infty} \mathrm{e}^{-\sigma t}|f(t)| \mathrm{d} t<+\infty\right\} \\
\text { and } \sigma_{\mathrm{u}}:=\sup \left\{\sigma \in \mathbb{R}: \int_{-\infty}^{\infty} \mathrm{e}^{-\sigma t}|f(t)| \mathrm{d} t<+\infty\right\},
\end{gathered}
$$

then the ROAC could be an open or closed interval that includes $\left(\sigma_{1}, \sigma_{\mathrm{u}}\right)$. For simplicity, in this paper we neglect the endpoints of the $\operatorname{ROAC}$, and require $\operatorname{Re}(s) \in\left(\sigma_{1}, \sigma_{\mathrm{u}}\right)$.

It is worth pointing out that the same two-sided Laplace transforms with different ROACs may correspond to different original functions; for example, consider three functions:

$$
\begin{aligned}
& f_{1}(t)= \begin{cases}\mathrm{e}^{2 t}-\mathrm{e}^{-3 t}, & t \geq 0, \\
0, & t<0,\end{cases} \\
& f_{2}(t)= \begin{cases}-\mathrm{e}^{-3 t}, & t \geq 0, \\
-\mathrm{e}^{2 t}, & t<0,\end{cases} \\
& f_{3}(t)= \begin{cases}0, & t \geq 0, \\
\mathrm{e}^{-3 t}-\mathrm{e}^{2 t}, & t<0 .\end{cases}
\end{aligned}
$$

It is easy to verify that their Laplace transforms are all $L(s)=5 /\left(s^{2}+s-6\right)$ but with different ROACs, $(2,+\infty),(-3,2)$, and $(-\infty,-3)$, respectively. Consequently, when inverting a twosided Laplace transform $L_{f}(s)$, we should first specify a particular ROAC. Moreover, the examples in (2.2) imply that the ROAC of a two-sided Laplace transform may not include the imaginary axis $\{s: \operatorname{Re}(s)=0\}$.

\subsection{Comparison with one-sided Laplace transforms and Fourier transforms}

If $f(t)=0$ for any $t<0$ then its two-sided Laplace transform is reduced to

$$
L_{f}(s)=\int_{0}^{+\infty} \mathrm{e}^{-s t} f(t) \mathrm{d} t \quad \text { for } \operatorname{Re}(\mathrm{s}) \in \operatorname{ROAC},
$$

and we call (2.3) the one-sided Laplace transform. The Fourier transform $\mathcal{F}_{f}(\omega): \mathbb{R} \mapsto \mathbb{C}$ of the function $f(t)$ is defined by

$$
\mathcal{F}_{f}(\omega):=\int_{-\infty}^{\infty} \mathrm{e}^{-\mathrm{i} \omega t} f(t) \mathrm{d} t \equiv L_{f}(\mathrm{i} \omega) \quad \text { for any } \omega \in \mathbb{R} .
$$

Because the ROAC of a two-sided Laplace transform may not include the imaginary axis $\{s: \operatorname{Re}(s)=0\}$, the Fourier transform of a function may not exist. For example, the Fourier transforms of $f_{1}(x)$ and $f_{3}(x)$ in (2.2) do not exist, whereas their two-sided Laplace transforms are well defined. Therefore, the Fourier transform is a special case of the two-sided Laplace transform.

\section{The two-sided Laplace inversion formula}

Our two-sided Laplace inversion formula involves parameters $C$ and $N$ for the purpose of controlling the discretization and truncation errors, respectively. 
Assumption 3.1. The function $\mathrm{e}^{-\sigma t} f(t)$ is of bounded variation on $\mathbb{R}$ for any $\sigma \in R O A C$.

Theorem 3.1. Consider a function $f(t)$ normalized such that $2 f(t)=f(t+0)+f(t-0)$ for any real $t$, where $f(t \pm 0):=\lim _{\varepsilon \downarrow} f(t \pm \varepsilon)$. Then, under Assumption 3.1, for any $t$ and $\sigma \in R O A C$,

$$
f(t)=f_{A}(t, \sigma, C, N)+e_{\mathrm{T}}(t, \sigma, C, N)-e_{\mathrm{D}}(t, \sigma, C),
$$

where the output of the inversion algorithm is

$$
\begin{aligned}
f_{A}(t, \sigma, C, N):= & \frac{\mathrm{e}^{\sigma t} L_{f}(\sigma)}{2(|t|+C)} \\
& +\frac{\mathrm{e}^{\sigma t}}{|t|+C} \sum_{k=1}^{N}\left[( - 1 ) ^ { k } \operatorname { R e } \left(\exp \left\{-\frac{\operatorname{sgn}(t) C k \pi \mathrm{i}}{t+\operatorname{sgn}(t) C}\right\}\right.\right. \\
& \left.\left.\times L_{f}\left(\sigma+\frac{k \pi \mathrm{i}}{t+\operatorname{sgn}(t) C}\right)\right)\right],
\end{aligned}
$$

$C \geq 0$ is a constant such that $|t|+C \neq 0, N>0$ is a positive integer, and $\operatorname{sgn}(x)$ equals 1 if $x \geq 0$ and equals -1 otherwise. The terms $e_{\mathrm{T}}(t, \sigma, C, N)$ and $e_{\mathrm{D}}(t, \sigma, C)$ represent the truncation error and the discretization error, respectively:

$$
\begin{aligned}
e_{\mathrm{T}}(t, \sigma, C, N):=\frac{\mathrm{e}^{\sigma t}}{|t|+C} \sum_{k=N+1}^{+\infty}\left[(-1)^{k} \operatorname{Re}(\right. & \exp \left\{-\frac{\operatorname{sgn}(t) C k \pi \mathrm{i}}{t+\operatorname{sgn}(t) C}\right\} \\
& \left.\left.\times L_{f}\left(\sigma+\frac{k \pi \mathrm{i}}{t+\operatorname{sgn}(t) C}\right)\right)\right]
\end{aligned}
$$

and

$$
e_{\mathrm{D}}(t, \sigma, C):=\sum_{k=-\infty, k \neq 0}^{+\infty} \mathrm{e}^{-2 \sigma k(t+\operatorname{sgn}(t) C)} f(2 k(t+\operatorname{sgn}(t) C)+t) .
$$

Proof. See Appendix A.

A sufficient condition for Assumption 3.1 to hold is given as follows.

Lemma 3.1. Consider a function $f(x) \in \mathcal{C}^{1}$. If there exist a constant $c$ and a monotone function $\bar{f}(x)$ such that $f(x)=\mathrm{e}^{c x} \bar{f}(x)$, then Assumption 3.1 holds.

Proof. See Appendix A.

\section{Discretization errors}

\subsection{Exponential decay of discretization errors}

In practice, one chooses a closed interval $\left[\sigma_{1}^{*}, \sigma_{\mathrm{u}}^{*}\right] \subset$ ROAC to do the numerical inversion. Without loss of generality, we assume that $\sigma_{1}^{*} \sigma_{\mathrm{u}}^{*} \neq 0$, and that $\sigma_{1}^{*}$ and $\sigma_{\mathrm{u}}^{*}$ are both finite. The following theorem shows that introducing the discretization parameter $C$ can make the discretization error decay exponentially for $\sigma \in\left(\sigma_{1}^{*}, \sigma_{\mathrm{u}}^{*}\right)$.

Theorem 4.1. If there exists a nonnegative function $\delta(\cdot)$ such that, for any $\sigma \in\left[\sigma_{1}^{*}, \sigma_{\mathrm{u}}^{*}\right]$, we have

$$
\mathrm{e}^{-\sigma y}|f(y)| \leq \delta(\sigma)<+\infty \text { for any } y
$$


then, for any fixed $t \in \mathbb{R}, \sigma \in\left(\sigma_{1}^{*}, \sigma_{\mathrm{u}}^{*}\right)$, and $C>0$, we have the error bound

$$
\left|e_{\mathrm{D}}(t, \sigma, C)\right| \leq \frac{\rho(\sigma, t)}{\mathrm{e}^{\theta(\sigma) C}-1},
$$

where $\theta(\sigma):=2 \min \left\{\sigma_{\mathrm{u}}^{*}-\sigma, \sigma-\sigma_{1}^{*}\right\}>0$ and

$$
\rho(\sigma, t):= \begin{cases}\delta\left(\sigma_{\mathrm{u}}^{*}\right) \mathrm{e}^{\left(2 \sigma-\sigma_{\mathrm{u}}^{*}\right) t}+\delta\left(\sigma_{1}^{*}\right) \mathrm{e}^{\left(3 \sigma_{1}^{*}-2 \sigma\right) t} & \text { if } t \geq 0, \\ \delta\left(\sigma_{1}^{*}\right) \mathrm{e}^{\left(2 \sigma-\sigma_{1}^{*}\right) t}+\delta\left(\sigma_{\mathrm{u}}^{*}\right) \mathrm{e}^{\left(3 \sigma_{\mathrm{u}}^{*}-2 \sigma\right) t} & \text { if } t<0 .\end{cases}
$$

Proof. See Appendix B.

In many applications, the upper bound (4.2) of the discretization error is computable because $\delta(\sigma)$ can be specified explicitly (see Sections 6.2 and 7.2). This enables us to easily choose a sufficiently large $C$ to control the discretization error.

Although numerical results show that the discretization error bound (4.2) could be very small, it seems quite difficult to analyze how tight the discretization error bound is with respect to the 'true' discretization error because the 'true' discretization error given in (3.3) involves the original function $f(\cdot)$ and is hence unknown. On the other hand, from Theorem 4.1 we can see that the discretization error bound depends heavily on the function $\delta(\sigma)$, while $\delta(\sigma)$ is specified case by case and could be selected in different ways even in the same case. Therefore, the tightness of the discretization error bound also relies on the selection of $\delta(\sigma)$ and, hence, varies from case to case. However, although it is unclear how tight the discretization error bound is, numerical examples in our paper suggest that we can always choose a sufficiently large $C$ based on this discretization error bound such that the discretization error is small enough to achieve the desired accuracy (Figure 3 in Section 8 illustrates that sometimes the discretization error bound is quite tight).

\subsection{Necessity of introducing the discretization parameter $C$}

We introduce the discretization parameter $C$ primarily for the purpose of controlling the discretization error. In the two-sided case, without $C$, the discretization error is given by $\sum_{k=-\infty, k \neq 0}^{+\infty} \mathrm{e}^{-2 \sigma k t} f((2 k+1) t)$, and is difficult to control; for example, if $\sigma>0(\sigma \leq$ 0 , respectively), the coefficients $\mathrm{e}^{-2 \sigma k t}$ for $k \leq-1$ ( $k \geq 1$, respectively) might be large. Example 4.1 illustrates this difficulty clearly.

Example 4.1. Consider the PDF of a standard normal distribution

$$
f(t)=\frac{1}{\sqrt{2 \pi}} \mathrm{e}^{-t^{2} / 2}
$$

with Laplace transform

$$
L_{f}(s)=\mathrm{e}^{s^{2} / 2} \quad \text { for } \sigma \equiv \operatorname{Re}(s) \in \mathbb{R}
$$

Assumption 3.1 holds because, for any $\sigma \in \mathbb{R}, \mathrm{e}^{-\sigma t} f(t)$ is in $\mathrm{C}^{1}$ and $\int_{-\infty}^{+\infty}\left|\left(\mathrm{e}^{-\sigma t} f(t)\right)^{\prime}\right| \mathrm{d} t<$ $+\infty$. Let us evaluate $f(t)$ at $t_{0}=0.5$ by inverting its Laplace transform $L_{f}(s)$ via the twosided Laplace inversion algorithm. If $C=0$, i.e. without the discretization parameter, the discretization error satisfies

$$
e_{\mathrm{D}}(0.5, \sigma, 0) \geq \begin{cases}f(-0.5) \mathrm{e}^{\sigma} \geq f(-0.5) \approx 0.35207 & \text { if } \sigma>0 \\ f(1.5) \mathrm{e}^{-\sigma} \geq f(1.5) \approx 0.12952 & \text { if } \sigma \leq 0\end{cases}
$$




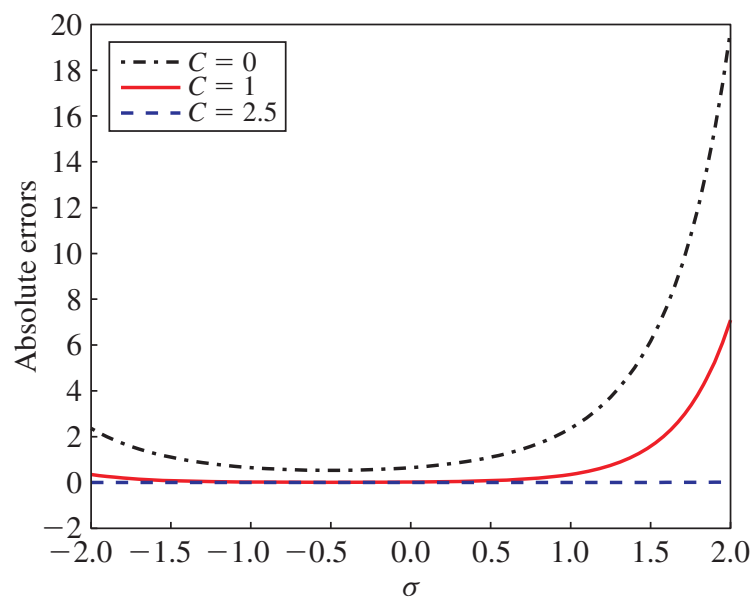

FiguRe 1: Comparison of absolute errors when evaluating the standard normal density at 0.5 via the twosided Laplace inversion algorithm with $C=0,1$, and 2.5 and $N=1000$. When $C=0$, i.e. without the discretization parameter, the absolute errors are always large (the minimum absolute error is 0.53043 ), no matter what $\sigma$ is chosen in [-2,2]. In comparison, when $C$ increases from 0 to 1 and then to 2.5 , the absolute error decreases significantly.

In other words, applying the Laplace inversion algorithm with $C=0$ would lead to large discretization errors, no matter what $\sigma$ is selected. This is also verified numerically in Figure 1 , where we can see that the absolute errors are always large (the minimum absolute error is 0.53043 ) when $\sigma$ varies in $[-2,2]$. In comparison, when $C$ increases from 0 to 1 and then to 2.5 , the absolute error decreases significantly.

\subsection{Comparison with Petrella's (2004) two-sided Laplace inversion algorithm}

The above example shows that it is necessary to introduce $C$ in some cases. Otherwise, the discretization error can always be large, no matter what $\sigma$ is chosen. On the other hand, Theorem 4.1 implies that $C$ should be sufficiently large to achieve a desired accuracy.

Petrella [14] numerically inverted the two-sided Laplace transform, i.e. evaluated the contour integral, along a specific vertical line:

$$
\sigma=\operatorname{Re}(s)=\frac{A}{2(t-C)}, \quad \text { where } A>0 \text { is a constant and typically set to be, e.g. } 20 .
$$

(He used the notation $\log X$ instead of $C$.) Nonetheless, this imposes a constraint on $C$ because $A / 2(t-C)$ should fall in the ROAC; as a result, $C$ might not be large enough to achieve the desired accuracy. See Example 4.2.

Example 4.2. Consider the function

$$
f(t)=\left(\mathrm{e}^{2 t}-\mathrm{e}^{-3 t}\right) \mathbf{1}_{\{t \geq 0\}},
$$

with Laplace transform

$$
L_{f}(s)=\frac{5}{(s-2)(s+3)} \quad \text { for } \sigma \equiv \operatorname{Re}(s) \in(2,+\infty) .
$$



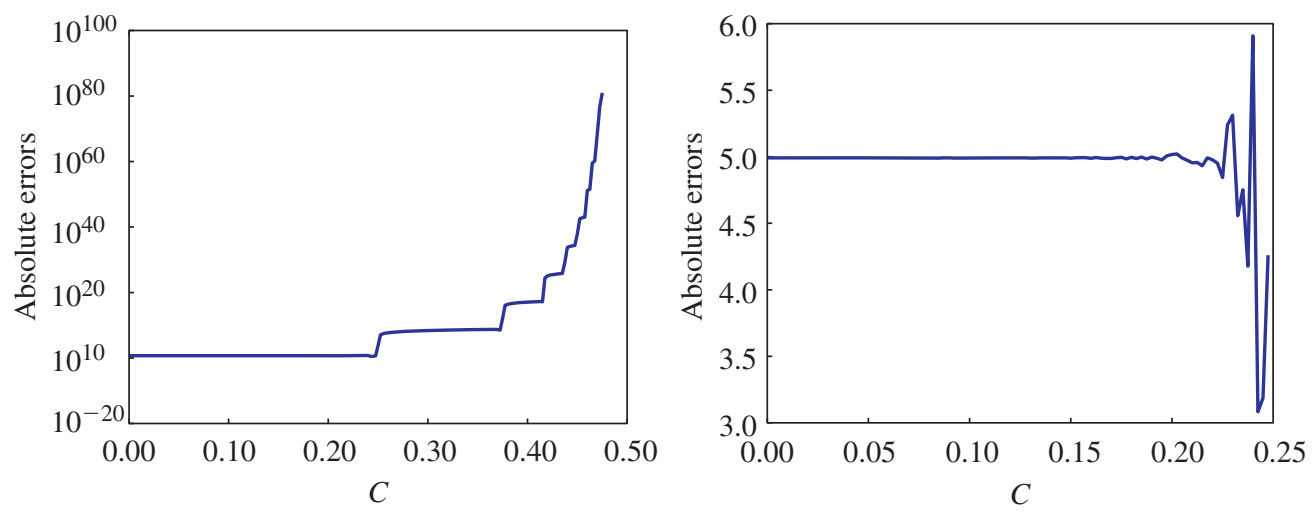

FIGURE 2: Absolute errors of numerical results when evaluating $f(t)=\left(\mathrm{e}^{2 t}-\mathrm{e}^{-3 t}\right) \mathbf{1}_{\{t \geq 0\}}$ at 0.5 via Pertrella's method ( $A=20$ and $N=10000)$. The right-hand plot is a magnified view of the region $[0,0.2475]$ of the left-hand plot. This figure demonstrates that, for any $C \in[0,0.5)$, Petrella's method leads to a large discretization error. Indeed, the minimum absolute error is approximately 3.08 and the error tends to explode (over $10^{80}$ ) as $C$ approaches 0.5 .

Assumption 3.1 holds because, for any $\sigma>2, \mathrm{e}^{-\sigma t} f(t)=\left[\mathrm{e}^{-(\sigma-2) t}-1\right] \mathbf{1}_{\{t \geq 0\}}-\left[\mathrm{e}^{-(\sigma+3) t}-\right.$ $1] \mathbf{1}_{\{t \geq 0\}}$ is the difference of two bounded nonincreasing functions. Let us evaluate $f(t)$ at $t_{0}=0.5$ by inverting its Laplace transform $L_{f}(s)$ through Pertrella's method. It is required that $A / 2\left(t_{0}-C\right) \in(2,+\infty)$. Assume that $A=20$. Then the constraint becomes $0 \leq C<0.5$. Figure 2 illustrates that, for any $C \in[0,0.5)$, Petrella's method leads to a large discretization error. Indeed, the minimum error is approximately 3.08 and the error tends to explode (over $10^{80}$ ) as $C$ approaches 0.5 .

In contrast, our algorithm imposes no constraints on $C$. As long as $\sigma \in(2,+\infty)$ (namely in the ROAC), selecting sufficiently large $C$ and $N$ and inverting $L_{f}(s)$ along the vertical line $\operatorname{Re}(s)=\sigma$ generates accurate numerical results. For example, choosing $\sigma=3, C=8$, and $N=10000$ leads to an accurate numerical result with an absolute error approximately $1.7 \times 10^{-7}$.

\section{Truncation errors}

In addition to the discretization error $e_{\mathrm{D}}(t, \sigma, C)$, the two-sided Laplace inversion formula (3.1) also contains the truncation error $e_{\mathrm{T}}(t, \sigma, C, N)$. Theorem 5.1 below gives upper bounds for the truncation error when the Laplace transform satisfies certain asymptotic conditions that often hold in financial engineering.

Theorem 5.1. For any fixed $t \in \mathbb{R}, \sigma \in R O A C$, and $C \geq 0$ such that $|t|+C>0$, the following statements hold.

(i) If there exist $\rho>0, \omega^{*} \geq 0$, and $\zeta(\sigma)>0$ such that

$$
\left|L_{f}(\sigma+\mathrm{i} \omega)\right| \leq \zeta(\sigma)|\omega|^{-(1+\rho)} \text { for all }|\omega|>\omega^{*},
$$

then the truncation error

$$
\left|e_{\mathrm{T}}(t, \sigma, C, N)\right| \leq \frac{\zeta(\sigma) \mathrm{e}^{\sigma t}(|t|+C)^{\rho}}{\rho \pi^{1+\rho}} N^{-\rho}=O\left(N^{-\rho}\right)
$$

for any $N \in \mathbb{N}$ such that $N>(|t|+C) \omega^{*} / \pi-1$. 
(ii) If there exist $\beta \in \mathbb{R}, \xi>0, \rho>0, \omega^{*} \geq 0$, and $\zeta(\sigma)>0$ such that

$$
\left|L_{f}(\sigma+\mathrm{i} \omega)\right| \leq \zeta(\sigma)|\omega|^{-\beta} \mathrm{e}^{-\rho|\omega|^{\xi}} \quad \text { for all }|\omega|>\omega^{*},
$$

then the truncation error

$$
\left|e_{\mathrm{T}}(t, \sigma, C, N)\right| \leq \frac{\zeta(\sigma) \mathrm{e}^{\sigma t}}{\pi \xi \rho^{(1-\beta) / \xi}} \Gamma\left(\frac{1-\beta}{\xi}, \rho \alpha N^{\xi}\right)=O\left(N^{1-\beta-\xi} \mathrm{e}^{-\rho \alpha N^{\xi}}\right)
$$

for any $N \in \mathbb{N}$ such that $N>(|t|+C) \omega^{*} / \pi-1$. Here $\alpha:=(\pi /(|t|+C))^{\xi}>0$, and, for any $s \in \mathbb{R}, \Gamma(s, x):=\int_{x}^{+\infty} y^{s-1} \mathrm{e}^{-y} \mathrm{~d} y$ denotes the upper incomplete gamma function.

Proof. See Appendix C.

In many applications, the upper bounds (5.2) and (5.4) of the truncation error are computable because the parameters in (5.1) and (5.3) can be identified explicitly (see Sections 6.3 and 6.4). Thus, for any $C>0$, we can easily specify a sufficiently large $N$ to control the truncation error.

In summary, the two-sided Laplace inversion algorithm can be implemented to achieve any desired accuracy by controlling both discretization and truncation errors. For example, if the desired accuracy is $10^{-5}$, we can take the following two steps to achieve it.

Step 1. Select a sufficiently large $C$ such that the discretization error is below $5 \times 10^{-6}$.

Step 2. For the fixed $C$ selected in step 1 , choose a sufficiently large $N$ such that the truncation error is below $5 \times 10^{-6}$.

Note that the selection of $\sigma$ also affects the selection of $C$ and $N$ (in a complicated way) because $\sigma$ is involved in the discretization error bound (4.2) and the truncation error bound (5.2) or (5.4). From (4.2), we can see that $\sigma$ affects the selection of $C$ through $\rho(\sigma, t)$ (where the function $\delta(\sigma)$ is involved) and $\mathrm{e}^{\theta(\sigma) C}$. However, $\delta(\sigma)$ is specified case by case and could be selected in different ways even in the same case. Therefore, the effect of $\sigma$ on the selection of $C$ also depends on the selection of $\delta(\sigma)$ and might need to be analyzed case by case. Similarly, from (5.2) or (5.4), it can be seen that $\sigma$ affects the selection of $N$ through $\zeta(\sigma) \mathrm{e}^{\sigma t}$ as well as through $(|t|+C)^{\rho}$ (in the case of (5.2), because $\sigma$ affects the selection of $C$ ) or $(\pi /(|t|+C))^{\xi}$ (in the case of (5.4)). Nonetheless, the function $\zeta(\sigma)$ and the values of $\rho$ and $\xi$ are different if the quantities to compute and/or the models are different. Accordingly, the effect of $\sigma$ on the selection of $N$ also relies on $\zeta(\sigma), \rho$, and $\xi$, and might need to be analyzed case by case.

It is worth pointing out that, although the value of $\sigma$ affects the selection of $C$ and $N$, whatever $\sigma$ is selected, we can always use the discretization and truncation error bounds to achieve the desired accuracy by choosing sufficiently large $C$ and $N$. Moreover, numerical examples suggest that the resulting algorithms are accurate and fast.

\section{Applications in financial engineering}

Two-sided Laplace transforms have been widely used in financial engineering. For example, analytical pricing formulae may not be available for European options under many asset pricing models, whereas their Laplace transforms with respect to (w.r.t.) $k:=-\log K$ often have closed-form expressions, where $K$ is the strike price. These Laplace transforms are two-sided because $k \in \mathbb{R}$. In this section we focus on the valuation of European options via Laplace inversion, whereas in the next section we demonstrate its application in exotic option pricing. 
TABLE 1: Connections among some Laplace transforms in financial engineering.

\begin{tabular}{cccc}
\hline & Original functions & Laplace transforms & ROAC \\
\hline PDF & $f_{X_{t}}(x)$ & $L_{f_{X_{t}}}(s)$ & $\left(\sigma_{1}, \sigma_{\mathrm{u}}\right)$ \\
$\mathrm{CDF}$ & $F_{X_{t}}(x):=\mathbb{P}\left(X_{t} \leq x\right)$ & $L_{F_{X_{t}}}(s)=\frac{L_{f_{X_{t}}}(s)}{s}$ & $\left(0, \sigma_{\mathrm{u}}\right)$ \\
$\begin{array}{c}\text { European call } \\
\text { option price }\end{array}$ & $\mathrm{EuC}(k):=\mathrm{e}^{-r t} \mathbb{E}\left[\left(S_{t}-\mathrm{e}^{-k}\right)^{+}\right]$ & $L_{\mathrm{EuC}}(s)=\frac{\mathrm{e}^{-r t} S_{0}^{s+1}}{s(s+1)} L_{f_{X_{t}}}(-s-1)$ & $\left(0,-\sigma_{1}-1\right)$ \\
\hline
\end{tabular}

\subsection{Laplace transforms}

Consider an asset pricing model $\left\{S_{t}=S_{0} \mathrm{e}^{X_{t}}: t \geq 0\right\}$ under a risk-neutral measure $\mathbb{P}$, where $\left\{X_{t}: t \geq 0\right\}$ denotes the asset return process. Assume that, for any fixed $t \geq 0, X_{t}$ has a continuous distribution, and the Laplace transform of the PDF $f_{X_{t}}(x)$ of $X_{t}$ is

$$
L_{f_{X_{t}}}(s)=\int_{-\infty}^{+\infty} \mathrm{e}^{-s x} f_{X_{t}}(x) \mathrm{d} x=\mathbb{E}^{-s X_{t}} \quad \text { for any } \operatorname{Re}(s) \in\left(\sigma_{1}, \sigma_{\mathrm{u}}\right) .
$$

Assume that $\sigma_{1}<-1$ and $\sigma_{\mathrm{u}}>0$. Then the Laplace transform of the CDF $F_{X_{t}}(x)$ w.r.t. $x$ and that of the European call option price $\operatorname{EuC}(k)$ w.r.t. $k$ can be expressed in terms of $L_{f_{X_{t}}}(s)$ (see Table 1).

Proposition 6.1 below shows that, under mild conditions, the CDF $F_{X_{t}}(x)$ and the European call option price $\operatorname{EuC}(k)$ satisfy Assumption 3.1. Accordingly, the two-sided Laplace inversion algorithm applies. If the Laplace transform $L_{f}(\sigma+\mathrm{i} \omega)$ of $X_{t}$ satisfies (5.1) or (5.3), then the two-sided Laplace inversion formula also applies for computing the PDF (see [5]).

Proposition 6.1. (i) If $X_{t}$ has a continuous distribution under the risk-neutral measure $\mathbb{P}$, then $\mathrm{e}^{-\sigma x} F_{X_{t}}(x)$ is of bounded variation on $\mathbb{R}$ for any $\sigma \in\left(0, \sigma_{\mathrm{u}}\right)$.

(ii) If $X_{t}$ has a continuous distribution under both the risk-neutral measure $\mathbb{P}$ and the numéraire measure $\widetilde{\mathbb{P}}\left(\right.$ namely, $\left.\mathrm{d} \widetilde{\mathbb{P}} /\left.\mathrm{d} \mathbb{P}\right|_{\mathcal{F}_{t}}=\mathrm{e}^{X_{t}-r t}\right)$, then $\mathrm{e}^{-\sigma k} E u C(k)$ is of bounded variation on $\mathbb{R}$ for any $\sigma \in\left(0,-\sigma_{1}-1\right)$.

Proof. See Appendix A of the electronic companion at [4].

\subsection{Discretization errors}

We need to identify $\delta(\sigma)$ in (4.1) when applying Theorem 4.1 for the discretization error. One particular specification is given in Table 2. The result of the CDF follows because

$$
\mathrm{e}^{-\sigma y} \mathbb{P}\left(X_{t} \leq y\right) \leq \mathrm{e}^{-\sigma y} \frac{\mathbb{E}\left[\mathrm{e}^{-\sigma X_{t}}\right]}{\mathrm{e}^{-\sigma y}}=L_{f_{X_{t}}}(\sigma) .
$$

The result of the European call option price follows because

$$
\begin{aligned}
\mathrm{e}^{-\sigma y} \mathbb{E}\left[S_{t} \mathbf{1}_{\left\{S_{t} \geq \mathrm{e}^{-y}\right\}}\right] & \leq \mathrm{e}^{-\sigma y}\left[\mathbb{E}\left(S_{t}^{p}\right)\right]^{1 / p}\left[\mathbb{P}\left(S_{t} \geq \mathrm{e}^{-y}\right)\right]^{1 / q} \\
& \leq \mathrm{e}^{-\sigma y}\left[\mathbb{E}\left(S_{t}^{p}\right)\right]^{1 / p}\left[\frac{\mathbb{E}\left(S_{t}^{q \sigma}\right)}{\mathrm{e}^{-q \sigma y}}\right]^{1 / q} \\
& =S_{0}^{\sigma+1}\left[L_{f_{X_{t}}}(-p)\right]^{1 / p}\left[L_{f_{X_{t}}}(-q \sigma)\right]^{1 / q},
\end{aligned}
$$


TABLE 2: Specification of $\delta(\sigma)$ in (4.1) for the upper bounds of the discretization error. Here $p=$ $\sigma_{1}\left(1-\sigma_{1}-\sigma_{\mathrm{u}}^{*}\right) / 2\left(\sigma_{1}+\sigma_{\mathrm{u}}^{*}\right)>1$.

\begin{tabular}{cc}
\hline $\mathrm{CDF}$ & $\delta(\sigma)$ in $(4.1)$ \\
\hline $\begin{array}{c}\text { European call } \\
\text { option price }\end{array}$ & $\mathrm{e}^{-r t} S_{0}^{\sigma+1}\left\{\left[L_{f_{X_{t}}}(-p)\right]^{1 / p}\left[L_{f_{X_{t}}}\left(-\frac{p \sigma}{p-1}\right)\right]^{1-1 / p}+L_{f_{X_{t}}}(-\sigma-1)\right\}$ \\
\hline
\end{tabular}

where the first inequality follows from Hölder's inequality with $1 / p+1 / q=1$ and $p, q>1$. For $\sigma \in\left[\sigma_{1}^{*}, \sigma_{\mathrm{u}}^{*}\right] \subset\left(0,-\sigma_{1}-1\right), \sigma_{1}<-1$, and $\sigma_{\mathrm{u}}>0$, it is required that

$$
\begin{gathered}
p>1, \quad-p \in\left(\sigma_{1}, \sigma_{\mathrm{u}}\right) \quad \text { and } \quad-q \sigma=-\frac{p \sigma}{p-1} \in\left(\sigma_{1}, \sigma_{\mathrm{u}}\right) \\
\Longleftrightarrow p \in\left(1,-\sigma_{1}\right) \text { and } p>\frac{\sigma_{1}}{\sigma_{1}+\sigma} .
\end{gathered}
$$

Since $1<\sigma_{1} /\left(\sigma_{1}+\sigma\right)<-\sigma_{1}$, it follows that we require

$$
p \in\left(\frac{\sigma_{1}}{\sigma_{1}+\sigma},-\sigma_{1}\right) \quad \text { for any } \sigma \in\left[\sigma_{1}^{*}, \sigma_{\mathrm{u}}^{*}\right] \quad \Longleftrightarrow p \in\left(\frac{\sigma_{1}}{\sigma_{1}+\sigma_{\mathrm{u}}^{*}},-\sigma_{1}\right) \text {. }
$$

Therefore, one particular choice of $p$ is the middle point $\sigma_{1}\left(1-\sigma_{1}-\sigma_{\mathrm{u}}^{*}\right) /\left(2\left(\sigma_{1}+\sigma_{\mathrm{u}}^{*}\right)\right)>1$.

If the objective is to compute a PDF $f_{X_{t}}(\cdot)$, the probabilistic method above used to identify the function $\delta(\sigma)$ for the CDF and European option price does not apply. One way to specify the function $\delta(\sigma)$ is as follows. We assume that its Laplace transform $L_{f}(\sigma+\mathrm{i} \omega)$ satisfies (5.1) or (5.3) (these conditions are required to derive the upper bound for the truncation error and often hold in financial engineering). By the Bromwich contour integral, we obtain, for any $\sigma \in \mathrm{ROAC}$,

$$
\begin{aligned}
\mathrm{e}^{-\sigma y}|f(y)| & =\frac{\mathrm{e}^{-\sigma y}}{2 \pi}\left|\int_{-\infty}^{+\infty} \mathrm{e}^{(\sigma+\mathrm{i} \omega) y} L_{f}(\sigma+\mathrm{i} \omega) \mathrm{d} \omega\right| \\
& \leq \frac{1}{2 \pi} \int_{-\infty}^{+\infty}\left|L_{f}(\sigma+\mathrm{i} \omega)\right| \mathrm{d} \omega \text { for any } y \in \mathbb{R} .
\end{aligned}
$$

Therefore, if (5.1) holds with $\omega^{*}>0$ (if $\omega^{*}=0$, we can replace $\omega^{*}$ by $\omega^{*}+1$ and the following argument still applies), we have

$$
\begin{aligned}
\mathrm{e}^{-\sigma y}|f(y)| \leq & \frac{1}{2 \pi} \int_{-\omega^{*}}^{\omega^{*}}\left|L_{f}(\sigma+\mathrm{i} \omega)\right| \mathrm{d} \omega+\frac{1}{2 \pi} \int_{\omega^{*}}^{+\infty}\left|L_{f}(\sigma+\mathrm{i} \omega)\right| \mathrm{d} \omega \\
& +\frac{1}{2 \pi} \int_{-\infty}^{-\omega^{*}}\left|L_{f}(\sigma+\mathrm{i} \omega)\right| \mathrm{d} \omega \\
= & \frac{1}{2 \pi} \int_{-\omega^{*}}^{\omega^{*}}\left|\int_{-\infty}^{+\infty} \mathrm{e}^{-(\sigma+\mathrm{i} \omega) x} f(x) \mathrm{d} x\right| \mathrm{d} \omega+\frac{1}{\pi} \int_{\omega^{*}}^{+\infty}\left|L_{f}(\sigma+\mathrm{i} \omega)\right| \mathrm{d} \omega \\
\leq & \frac{1}{2 \pi} \int_{-\omega^{*}}^{\omega^{*}} \int_{-\infty}^{+\infty} \mathrm{e}^{-\sigma x} f(x) \mathrm{d} x \mathrm{~d} \omega+\frac{1}{\pi} \int_{\omega^{*}}^{+\infty} \zeta(\sigma) \omega^{-(1+\rho)} \mathrm{d} \omega \\
= & \frac{\omega^{*}}{\pi} L_{f}(\sigma)+\frac{1}{\pi \rho \omega^{* \rho}} \zeta(\sigma) \\
< & +\infty
\end{aligned}
$$


Then we can define

$$
\delta(\sigma):=\frac{\omega^{*}}{\pi} L_{f}(\sigma)+\frac{1}{\pi \rho \omega^{* \rho}} \zeta(\sigma)<+\infty .
$$

Similarly, if (5.3) holds with $\omega^{*}>0$ or $\beta<1$ (otherwise, we can replace $\omega^{*}$ by $\omega^{*}+1$ ), it follows that

$$
\mathrm{e}^{-\sigma y}|f(y)| \leq \frac{\omega^{*}}{\pi} L_{f}(\sigma)+\frac{1}{\pi \xi} \rho^{(\beta-1) / \xi} \Gamma\left(\frac{1-\beta}{\xi}, \rho \omega^{* \xi}\right) \zeta(\sigma)=: \delta(\sigma)<+\infty .
$$

\subsection{Truncation errors}

To use Theorem 5.1 for the truncation error, we need to specify parameters in either (5.1) or (5.3). In Table 3 we provide an easy way to obtain these parameters. For example, if the Laplace transform of the PDF, $L_{f_{X_{t}}}(\sigma+\mathrm{i} \omega)$, satisfies (5.1) with parameters $\rho, \zeta(\sigma)$, and $\omega^{*}$, then the Laplace transform of the European call option price, $L_{\mathrm{EuC}}(\sigma+\mathrm{i} \omega)$, also satisfies $(5.1)$ with parameters $\rho+2, \mathrm{e}^{-r t} S_{0}^{\sigma+1} \zeta(-\sigma-1)$, and $\omega^{*}$, because, for any $|\omega|>\omega^{*}$,

$$
\begin{aligned}
\left|L_{\mathrm{EuC}}(\sigma+\mathrm{i} \omega)\right| & =\frac{\mathrm{e}^{-r t} S_{0}^{\sigma+1}}{\sqrt{\sigma^{2}+\omega^{2}} \sqrt{(\sigma+1)^{2}+\omega^{2}}}\left|L_{f_{X_{t}}}(-\sigma-1-\mathrm{i} \omega)\right| \\
& \leq \mathrm{e}^{-r t} S_{0}^{\sigma+1} \omega^{-2}\left|L_{f_{X_{t}}}(-\sigma-1-\mathrm{i} \omega)\right| .
\end{aligned}
$$

\subsection{Two examples}

6.4.1. The CGMY model. Under the CGMY model, the Laplace transform of the PDF $f_{X_{t}}(x)$ of the asset return $X_{t}$ is given by

$$
L_{f_{X_{t}}}(s)=\exp \left\{-\mu t s-t \hat{C} \Gamma(-\hat{Y})\left[\hat{M}^{\hat{Y}}-(\hat{M}+s)^{\hat{Y}}+\hat{G}^{\hat{Y}}-(\hat{G}-s)^{\hat{Y}}\right]\right\}
$$

for $\operatorname{Re}(s) \in(-\hat{M}, \hat{G})$, where

$$
\mu=r-q-\hat{C} \Gamma(-\hat{Y})\left[(\hat{M}-1)^{\hat{Y}}-\hat{M}^{\hat{Y}}+(\hat{G}+1)^{\hat{Y}}-\hat{G}^{\hat{Y}}\right],
$$

$\hat{C}>0, \hat{G}>0, \hat{M}>0, \hat{Y}<2$ but $\hat{Y} \neq 0$ and 1 , and $\Gamma(\cdot)$ represents the gamma function. Then the Laplace transform of the CDF $F_{X_{t}}(x)$ and that of the European call option price $\operatorname{EuC}(k)$ can be obtained immediately from Table 1 . According to Proposition 6.1, the two-sided Laplace inversion algorithm can be used.

The upper bounds of the discretization errors related to the CDF and the European call option price are given by Theorem 4.1, Table 2, and (6.1). The upper bounds of their truncation errors can be obtained by Theorem 5.1 with the parameters given in the following proposition.

TABLE 3: Connections among the parameters in (5.1) and (5.3) for the upper bounds of the truncation error.

\begin{tabular}{clll}
\hline & Laplace transforms & Parameters in (5.1) & Parameters in (5.3) \\
\hline $\mathrm{PDF}$ & $L_{f_{X_{t}}}(\sigma+\mathrm{i} \omega)$ & $\rho, \zeta(\sigma), \omega^{*}$ & $\rho, \beta, \xi, \zeta(\sigma), \omega^{*}$ \\
$\mathrm{CDF}$ & $L_{F_{X_{t}}}(\sigma+\mathrm{i} \omega)$ & $\rho+1, \zeta(\sigma), \omega^{*}$ & $\rho, \beta+1, \xi, \zeta(\sigma), \omega^{*}$ \\
European call & $L_{\mathrm{EuC}}(\sigma+\mathrm{i} \omega)$ & $\rho+2, \mathrm{e}^{-r t} S_{0}^{\sigma+1} \zeta(-\sigma-1), \omega^{*}$ & $\rho, \beta+2, \xi$, \\
option price & & & $\mathrm{e}^{-r t} S_{0}^{\sigma+1} \zeta(-\sigma-1), \omega^{*}$ \\
\hline
\end{tabular}


Proposition 6.2. The Laplace transform $L_{f_{X_{t}}}(\sigma+\mathrm{i} \omega)$ of the PDF $f_{X_{t}}(x)$ of a CGMY process $X_{t}$ satisfies (5.3) with the parameters

$$
\begin{gathered}
\zeta(\sigma)=\exp \left\{-\mu t \sigma-t \hat{C} \Gamma(-\hat{Y})\left(\hat{M}^{\hat{Y}}+\hat{G}^{\hat{Y}}\right)\right\}, \quad \beta=0, \quad \xi=\hat{Y}, \\
\rho=-2 t \hat{C} \Gamma(-\hat{Y}) \cos \left(\frac{\hat{Y} \pi}{2}\right) \quad \text { if } \hat{Y} \in(0,1), \\
\rho=-2 t \hat{C} \Gamma(-\hat{Y}) \cos \left(\frac{\pi}{2}+\varepsilon\right) \quad \text { if } \hat{Y} \in(1,2), \\
\omega^{*}=0 \quad \text { if } \hat{Y} \in(0,1), \quad \omega^{*}=\max \{\hat{M}+\sigma, \hat{G}-\sigma\} \tan \left(\frac{\pi / 2+\varepsilon}{\hat{Y}}\right) \quad \text { if } \hat{Y} \in(1,2),
\end{gathered}
$$

where $\varepsilon$ is any constant in $(0, \pi(\hat{Y}-1) / 2)$. The Laplace transforms of the CDF and the European call option price satisfy (5.3) with the parameters given in Table 3.

Proof. See Appendix B of the electronic companion [4].

6.4.2. The mixed-exponential jump diffusion model (MEM). Under the MEM (see [3]), the stock price $\left\{S_{t}: t \geq 0\right\}$ is given by

$$
S_{t}=S_{0} \mathrm{e}^{X_{t}} \quad \text { with the return process } X_{t}:=\mu t+\bar{\sigma} W_{t}+\sum_{j=1}^{N_{t}} Y_{j},
$$

where $\mu=r-\bar{\sigma}^{2} / 2-\lambda\left(\mathbb{E}^{Y_{1}}-1\right), r$ is the risk-free interest rate, $\bar{\sigma}>0$ is the volatility, $\left\{N_{t}\right\}$ is a Poisson process with rate $\lambda,\left\{W_{t}\right\}$ is a standard Brownian motion, and $\left\{Y_{j}: j=1,2, \ldots\right\}$ is a sequence of independent and identically distributed (i.i.d.) mixed-exponential random variables with the $\operatorname{PDF} f_{Y}(x)$ given by

$$
f_{Y}(x)=p_{u} \sum_{l=1}^{m} p_{l} \eta_{l} \mathrm{e}^{-\eta_{l} x} \mathbf{1}_{\{x \geq 0\}}+q_{d} \sum_{j=1}^{n} q_{j} \theta_{j} \mathrm{e}^{\theta_{j} x} \mathbf{1}_{\{x<0\}},
$$

where $p_{u} \geq 0, q_{d}=1-p_{u} \geq 0, p_{l} \in(-\infty, \infty)$ and $\eta_{l}>1$ for all $l, \sum_{l=1}^{m} p_{l}=1$, $q_{j} \in(-\infty, \infty)$ and $\theta_{j}>0$ for all $j$, and $\sum_{j=1}^{n} q_{j}=1$.

Without loss of generality, we assume that $\eta_{1}<\eta_{2}<\cdots<\eta_{m}$ and $\theta_{1}<\theta_{2}<\cdots<\theta_{n}$. The Laplace transform of the pdf $f_{X_{t}}(x)$ of $X_{t}$ is

$$
L_{f_{X_{t}}}(s)=\mathrm{e}^{G(-s) t} \quad \text { for } \operatorname{Re}(s) \in\left(\sigma_{1}, \sigma_{\mathrm{u}}\right) \equiv\left(-\eta_{1}, \theta_{1}\right),
$$

where $G(x)$ is defined as

$$
G(x)=\frac{\bar{\sigma}^{2}}{2} x^{2}+\mu x+\lambda\left(p_{u} \sum_{l=1}^{m} \frac{p_{l} \eta_{l}}{\eta_{l}-x}+q_{d} \sum_{j=1}^{n} \frac{q_{j} \theta_{j}}{\theta_{j}+x}-1\right) .
$$

Then the Laplace transform of the CDF $F_{X_{t}}(x)$ and that of the European call option price $\mathrm{EuC}(k)$ are given in Table 1. According to Proposition 6.1, the two-sided Laplace inversion algorithm applies.

The upper bounds of the discretization errors related to the CDF and the European call option price are given by Theorem 4.1, Table 2, and (6.2). The upper bounds of their truncation errors can be obtained by Theorem 5.1 with the parameters given in the following proposition. 
Proposition 6.3. The Laplace transform $L_{f_{X_{t}}}(\sigma+\mathrm{i} \omega)$ of the PDF $f_{X_{t}}(x)$ of the asset return $X_{t}$ under the MEM satisfies (5.3) with the parameters

$$
\begin{gathered}
\zeta(\sigma)=\exp \left\{t\left[\frac{\bar{\sigma}^{2}}{2} \sigma^{2}-\mu \sigma+\lambda\left(p_{u} \sum_{l=1}^{m} \frac{\left|p_{l}\right| \eta_{l}}{\eta_{l}+\sigma}+q_{d} \sum_{j=1}^{n} \frac{\left|q_{j}\right| \theta_{j}}{\theta_{j}-\sigma}-1\right)\right]\right\}, \\
\beta=0, \quad \rho=\frac{t \bar{\sigma}^{2}}{2}, \quad \xi=2, \quad \text { and } \quad \omega^{*}=0 .
\end{gathered}
$$

The Laplace transforms of the CDF and the European call option price satisfy (5.3) with the parameters given in Table 3.

Proof. See Appendix C of the electronic companion [4].

\section{Applications in financial engineering: pricing of exotic options}

In Section 6 we primarily discussed how to price vanilla options (and calculate related CDFs) via our two-sided Laplace inversion algorithm. In this section we demonstrate the application of our algorithm to the pricing of exotic options. We take the lookback option as an example, whose Laplace transform has a closed-form expression under the double-exponential jump diffusion model (DEM). Note that the DEM is a special case of the MEM with $m=n=1$. Throughout this section, we use the same notation as in Section 6.4.2 except that the parameters $p_{u} p_{1}, q_{d} q_{1}, \eta_{1}$ and $\theta_{1}$ are simplified as $p, q, \eta$, and $\theta$.

The price of a lookback put option under the risk-neutral measure $\mathbb{P}$ is given by

$$
L P(T)=\mathbb{E}\left[\mathrm{e}^{-r T}\left(\max \left\{M, \max _{0 \leq t \leq T} S_{t}\right\}-S_{T}\right)\right]=\mathbb{E}\left[\mathrm{e}^{-r T} \max \left\{M, \max _{0 \leq t \leq T} S_{t}\right\}\right]-S_{0},
$$

where $T>0$ is the maturity and $M>S_{0}$ is a fixed constant presenting a prescribed maximum at time 0. Under the DEM, Kou and Wang [11] obtained the Laplace transform of $L P(T)$ w.r.t. $T$ as

$$
\begin{aligned}
\int_{0}^{+\infty} \mathrm{e}^{-s T} L P(T) \mathrm{d} T= & M \frac{A_{s}}{C_{s}}\left(\frac{S_{0}}{M}\right)^{\beta_{1, s+r}}+M \frac{B_{s}}{C_{s}}\left(\frac{S_{0}}{M}\right)^{\beta_{2, s+r}} \\
& +\frac{M}{s+r}-\frac{S_{0}}{s} \quad \text { for } \operatorname{Re}(s)>0
\end{aligned}
$$

where

$$
A_{s}=\frac{\left(\eta-\beta_{1, s+r}\right) \beta_{2, s+r}}{\beta_{1, s+r}-1}, \quad B_{s}=\frac{\left(\beta_{2, s+r}-\eta\right) \beta_{1, s+r}}{\beta_{2, s+r}-1}, \quad C_{s}=\eta(s+r)\left(\beta_{2, s+r}-\beta_{1, s+r}\right),
$$

and $\beta_{1, s+r}$ and $\beta_{2, s+r}$ are the two roots with positive real parts of the equation $G(x)=s+r$.

To evaluate $L P(T)$ numerically, we will not invert its Laplace transform in (7.1) directly because this Laplace transform satisfies neither (5.1) nor (5.3) in Theorem 5.1. However, we find that it is simple to overcome this difficulty. Indeed, we can evaluate a new function $f(T)$ (the only difference between $f(T)$ and $L P(T)$ is a constant $S_{0}-M$ ),

$$
f(T):=\left[L P(T)-M+S_{0}\right] \mathbf{1}_{\{T \geq 0\}}=\left(\mathbb{E}\left[\mathrm{e}^{-r T} \max \left\{M, \max _{0 \leq t \leq T} S_{t}\right\}\right]-M\right) \mathbf{1}_{\{T \geq 0\}},
$$


instead by inverting its Laplace transform

$$
\begin{aligned}
L_{f}(s) & :=\int_{-\infty}^{+\infty} \mathrm{e}^{-s T} f(T) \mathrm{d} T \\
& =M \frac{A_{s}}{C_{s}}\left(\frac{S_{0}}{M}\right)^{\beta_{1, s+r}}+M \frac{B_{s}}{C_{s}}\left(\frac{S_{0}}{M}\right)^{\beta_{2, s+r}}+\frac{M}{s+r}-\frac{M}{s} \quad \text { for } \operatorname{Re}(s)>0 .
\end{aligned}
$$

Then the lookback option price is given by $L P(T)=f(T)+M-S_{0}$ for $T>0$. Note that $L_{f}(s)$ is a one-sided Laplace transform, a special case of two-sided Laplace transforms. Proposition 7.1 below shows that $f(T)$ satisfies Assumption 3.1. Therefore, our two-sided Laplace inversion algorithm applies.

Proposition 7.1. For any $\sigma>0$, the function $\mathrm{e}^{-\sigma T} f(T)$ is of bounded variation on $\mathbb{R}$.

Proof. See Appendix D of the electronic companion [4].

\subsection{Truncation errors}

To derive a computable truncation error bound using Theorem 5.1, it suffices to specify the asymptotic behavior of $\left|L_{f}(\sigma+\mathrm{i} \omega)\right|$; see Proposition 7.2 below.

Proposition 7.2. The Laplace transform $L_{f}(\sigma+\mathrm{i} \omega)$ of the function $f(T)=[L P(T)-M+$ $\left.S_{0}\right] \mathbf{1}_{\{T \geq 0\}}$ under the DEM satisfies (5.1) with the parameters

$$
\begin{gathered}
\zeta(\sigma)=r M+\frac{M Y_{3}}{\omega^{*} \theta \eta\left|\eta-Y_{3} / \omega^{*} \theta-1\right|} \frac{\left|z_{\omega^{*}}-\eta\right|-2|\mu| / \bar{\sigma}^{2}+\eta}{\left|z_{\omega^{*}}-\eta\right|-2|\mu| / \bar{\sigma}^{2}-Y_{3} / \omega^{*} \theta} \\
+\frac{10 \sqrt{2} \bar{\sigma} M}{b \mathrm{e}^{10}}\left(\frac{M}{S_{0}}\right)^{2|\mu| / \bar{\sigma}^{2}}\left(1+\frac{Y_{3}}{\omega^{*} \eta \theta}\right) \frac{\left|z_{\omega^{*}}-\eta\right|-2|\mu| / \bar{\sigma}^{2}}{\left|z_{\omega^{*}}-\eta\right|-2|\mu| / \bar{\sigma}^{2}-Y_{3} / \omega^{*} \theta}, \\
\rho=1, \quad \text { and } \omega^{*}=\max \left\{Y(\sigma+r), \frac{100}{b^{2}}\right\},
\end{gathered}
$$

where $b:=\log \left(M / S_{0}\right) / \bar{\sigma}>0, z_{y}:=\sqrt{|y|}(1+\mathrm{i} \cdot \operatorname{sgn}(y)) / \bar{\sigma}, Y(x):=\max \left\{Y_{1}, Y_{2}, Y_{3}, Y_{4}\right\}$, $Y_{1}=2(\bar{\sigma} \eta+2|\mu| / \bar{\sigma})^{2}, Y_{2}=\frac{1}{2}(4|\mu| / \bar{\sigma}+\bar{\sigma}(x+2 \lambda) /|\mu|)^{2}, Y_{3}=2 \eta^{2} \bar{\sigma}^{2}+2 \eta|\mu|+\lambda+x+$ $2 \lambda \eta|p \eta-q \theta|+\lambda \eta \theta$, and $Y_{4}=Y_{3} / \eta \theta$.

Proof. See Appendix D of the electronic companion [4].

\subsection{Discretization errors}

To derive a computable discretization error bound using Theorem 4.1, we need to identify $\delta(\sigma)$ in (4.1), which is given by Proposition 7.3 below.

Proposition 7.3. For any $\sigma>0$, we have

$$
\mathrm{e}^{-\sigma T}|f(T)| \leq \delta(\sigma):=\left(L_{f}(\sigma)+\frac{2 M}{\sigma}\right) \frac{\omega^{*}}{\pi}+\frac{\zeta(\sigma)}{\pi \omega^{*}},
$$

where $\omega^{*}$ and $\zeta(\sigma)$ are the same as in Proposition 7.2.

Proof. See Appendix D of the electronic companion [4]. 


\section{Numerical examples}

\subsection{Evaluating the CDF and pricing European call options under the CGMY model}

In Table 4 we provide the numerical results of the CDF $F_{X_{t}}\left(x_{j}\right)$ at $t=0.5$ for the three $x_{j}$ given by

$$
x_{j}=\mathbb{E}\left(X_{0.5}\right)+j \sqrt{\operatorname{var}\left(X_{0.5}\right)} \text { for } j=0 \text { and } \pm 10,
$$

as well as the upper bounds of both discretization and truncation errors. It seems that our numerical results are highly accurate even in the case of extreme $x$, e.g. $x_{10}$ and $x_{-10}$, and agree with those obtained by Feng and Lin [8] to 12 decimal points. Besides, it takes only 0.05 seconds to generate one numerical result via our method. All the computations in this paper are conducted using MATLAB ${ }^{\circledR} 7.1$ on a laptop with a $2.67 \mathrm{GHz}$ CPU.

Figure 3 gives the 'true' discretization errors and their upper bounds for various $C \in[2,6]$ (the left-hand panel), as well as the 'true' truncation errors and their upper bounds for various $N \in[36,196]$ (the right-hand panel), when evaluating $F_{X_{0.5}}\left(x_{0}\right)$ via the two-sided Laplace inversion algorithm. Here the 'true' discretization error is calculated as $f_{A}\left(t, \sigma, C, N^{*}\right)-$ $f_{A}\left(t, \sigma, C^{*}, N^{*}\right)$ and the 'true' truncation error is calculated as $f_{A}(t, \sigma, C, N)-f_{A}\left(t, \sigma, C, N^{*}\right)$, with $C^{*}=10$ and $N^{*}=20000$. We can see that the upper bounds of the discretization and truncation errors become very small when $C \geq 4$ and $N \geq 64$ (below $10^{-6}$ ), respectively, and moreover, the 'true' discretization and truncation errors are even smaller. Besides, it can be seen that in this example the actual discretization error is about one order of magnitude smaller than the discretization error bound, while the actual truncation error is about two orders of magnitude smaller than the truncation error bound.

Figure 4 provides the 'true' discretization errors, the 'true' truncation errors, and their upper bounds, when evaluating $F_{X_{0.5}}\left(x_{0}\right)$ via the two-sided Laplace inversion algorithm with different values of $\sigma(\sigma=1,2$, and 3). We can see that the selection of $\sigma$ does affect the selection of $C$ and $N$ (in this example, it has a bigger effect on the selection of $C$ ).

In Table 5 we provide numerical prices for European call options under the CGMY model via the two-sided Laplace inversion algorithm. The upper bounds of both discretization and truncation errors are also reported. It can be seen that our numerical prices are highly accurate when $K$ varies from 10 (deep in the money) to 200 (deep out of the money), and agree with those obtained by Feng and Lin [8] to 10 decimal points.

TABLE 4: The results in the column 'Feng \& Lin' are taken from Feng and Lin [8], while the results in the column 'Cai, Kou \& Liu' are derived via our two-sided Laplace inversion method. The model parameters are the same as in Feng and Lin [8], i.e. $\hat{C}=2, \hat{G}=5, \hat{M}=15, \hat{Y}=0.5, r=0.03, q=0$, and $t=0.5$. The algorithm parameters are $\sigma=2.5, \sigma_{1}^{*}=0.1, \sigma_{\mathrm{u}}^{*}=4.9, C=8$, and $N=350$. The upper bounds of both discretization and truncation errors are also reported. Here $x_{0}=-0.029, x_{10}=1.506$, and $x_{-10}=-3.099$, which are defined by (8.1). It takes only 0.05 seconds to generate one numerical result via our method.

\begin{tabular}{lcccc}
\hline \multicolumn{5}{c}{ Evaluating the CDF $F_{X_{t}}(x)$ of the CGMY process $X_{t}$ at $t=0.5$} \\
\hline & Feng \& Lin & Cai, Kou \& Liu & $\begin{array}{c}\text { Upper bounds of } \\
\text { discretization errors }\end{array}$ & $\begin{array}{c}\text { Upper bounds of } \\
\text { truncation errors }\end{array}$ \\
\hline$F_{X_{0.5}}\left(x_{-10}\right)$ & 0.000000152486 & 0.000000152486 & $5.4 \times 10^{-24}$ & $1.4 \times 10^{-18}$ \\
$F_{X_{0.5}}\left(x_{0}\right)$ & 0.450226233660 & 0.450226233660 & $4.2 \times 10^{-16}$ & $3.9 \times 10^{-19}$ \\
$F_{X_{0.5}}\left(x_{10}\right)$ & 0.999999976408 & 0.999999976408 & $6.1 \times 10^{-16}$ & $2.3 \times 10^{-15}$ \\
\hline
\end{tabular}



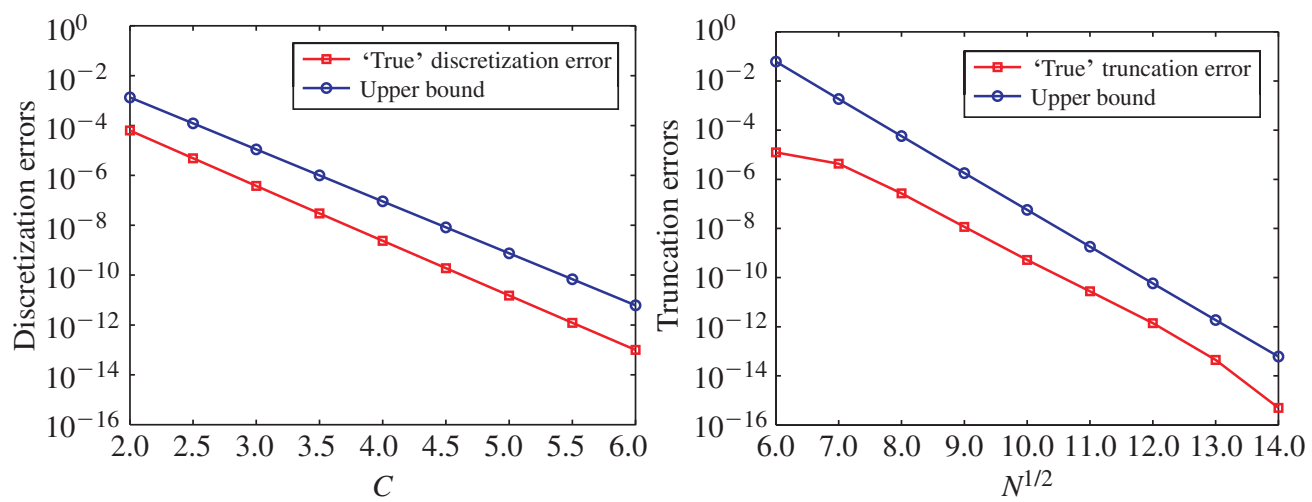

FIGURE 3: Discretization errors (left), truncation errors (right), and their upper bounds when evaluating the CDF $F_{X_{t}}\left(x_{0}\right)$ of the CGMY process $X_{t}$ at $t=0.5$ for $x_{0}=-0.029$, via the two-sided Laplace inversion algorithm. In calculating the 'true' errors, we set $N^{*}=20000$ and $C^{*}=10$. Other algorithm parameters are $\sigma=2.5, \sigma_{1}^{*}=0.1$, and $\sigma_{\mathrm{u}}^{*}=4.9$ (left), and $\sigma=2.5$ and $C=7$ (right). We can see that the upper bounds of the discretization and truncation errors become very small when $C \geq 4$ and $N \geq 64$ (below $10^{-6}$ ), respectively, and moreover, the 'true' discretization and truncation errors are even smaller.
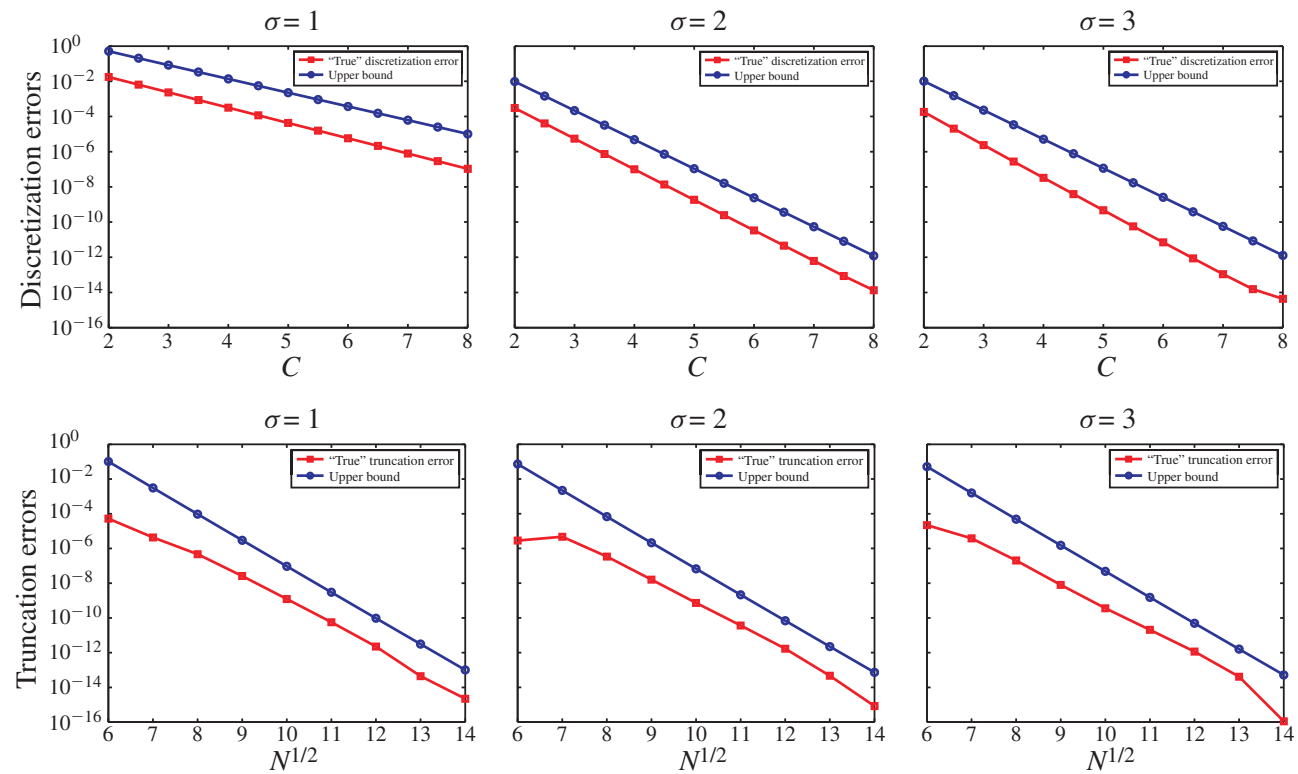

FIGURE 4: Discretization errors (top panels), truncation errors (bottom panels), and their upper bounds when evaluating the CDF $F_{X_{t}}\left(x_{0}\right)$ of the CGMY process $X_{t}$ at $t=0.5$ for $x_{0}=-0.029$, via the two-sided Laplace inversion algorithm with different values of $\sigma(\sigma=1,2$, and 3). Other parameters are the same as in Figure 3. 
TABLE 5: The model parameters are the same as in Carr and Madan [7], i.e. $S_{0}=100, \hat{C}=2, \hat{G}=5$, $\hat{M}=10, \hat{Y}=0.5, r=0.03, q=0$, and $t=0.5$. The algorithm parameters are $\sigma=2, \sigma_{1}^{*}=0.1$, $\sigma_{\mathrm{u}}^{*}=3.9, C=9$, and $N=350$. The upper bounds of both discretization and truncation errors are also reported. The column 'Feng \& Lin' is taken from [8]. The columns 'FFT' and 'SP' are taken from Carr and Madan [7], which are computed via the FFT method in [6] and the saddlepoint method in [7], respectively. It takes approximately 0.359 seconds to generate all the 20 prices via our algorithm.

\begin{tabular}{|c|c|c|c|c|c|}
\hline \multicolumn{6}{|c|}{ Pricing European call options under the CGMY model } \\
\hline$K$ Cai, Kou \& Liu & $\begin{array}{l}\text { Upper bounds } \\
\text { of discret. err. }\end{array}$ & $\begin{array}{l}\text { Upper bounds } \\
\text { of trunc. err. }\end{array}$ & Feng \& Lin & $\begin{array}{c}\text { FFT } \\
\text { (Abs. err.) }\end{array}$ & $\begin{array}{c}\text { SP } \\
\text { (Abs. err.) }\end{array}$ \\
\hline 1090.1488982036 & $8.9 \times 10^{-13}$ & $1.5 \times 10^{-13}$ & 90.1488982036 & 88.2148 & $90.1571(-0.0082)$ \\
\hline 2080.2990032439 & & & & $79.4972(\mathbf{0 . 8 0 1 8})$ & \\
\hline 3070.4611881539 & $1.9 \times 10$ & $1.8 \times 10$ & 70.461 & 561) & \\
\hline 4060.6764949331 & $2.1 \times$ & $1.8 \times 10^{-}$ & 60.676 & $60.6231(0.0534)$ & $397(-0.0632)$ \\
\hline 5051.0422031686 & $3.8 \times 10^{-18}$ & $1.8 \times 10^{-13}$ & 51.04220316 & 51.0359 & 51.0482 \\
\hline 6041.730704053 & & & & & 41.6 \\
\hline 7032.9873494847 & & & & & \\
\hline 8025.0978961195 & & $1.7 \times 10^{-}$ & & 27) & $24.7688(0.3291)$ \\
\hline 9018.3270683608 & & & & & $18.0184(\mathbf{0 . 3 0 8 7 )}$ \\
\hline 10012.8455624996 & $2.6 \times 10^{-20}$ & $1.6 \times 10^{-13}$ & 12.8455624996 & $12.8429(0.0027)$ & $12.6191(0.2265)$ \\
\hline 1108.676 & $1.4 \times 10^{-20}$ & $<10^{-13}$ & & & \\
\hline 1205.6918789863 & & & 89863 & 7) & $5.6278(0.0641)$ \\
\hline 1303.6627715757 & $5.2 \times 10^{-}$ & $1.5 \times 10^{-13}$ & 3.6627715757 & $3.6601(0.0027)$ & $3.6387(0.0241)$ \\
\hline 1402.3350436302 & & $1.5 \times 10^{-13}$ & 2.3350436302 & $2.3324(0.0026)$ & $2.3295(0.0055)$ \\
\hline 1501.4867227418 & $2.4 \times 10^{-21}$ & $1.4 \times 10^{-13}$ & 1.4867227418 & $1.4840(0.0027)$ & $1.4879(-0.0012)$ \\
\hline 1600 & & & & & \\
\hline 1700.6133 & $1.3 \times 10^{-21}$ & $1.4 \times 10^{-13}$ & & 2) & \\
\hline $\begin{array}{ll}180 & 0.3999541138\end{array}$ & $9.9 \times 10^{-22}$ & $1.3 \times 10^{-13}$ & 0.3999541138 & $0.3278(\mathbf{0 . 0 7 2 2})$ & $0.4018(-0.0018)$ \\
\hline $190 \quad 0.2639477786$ & & $1.3 \times 10^{-13}$ & 0.2639477786 & $0.0129(0.2510)$ & $0.2652(-0.0013)$ \\
\hline $200 \quad 0.1764092928$ & $6.2 \times 10^{-22}$ & $1.3 \times 10^{-13}$ & 0.1764092928 & $-0.4588(\boldsymbol{0 . 6 3 5 2})$ & $0.1772(-0.0008)$ \\
\hline
\end{tabular}

The columns 'FFT' and 'SP' are taken from Carr and Madan [7], which are computed through the fast Fourier transform (FFT) method in [6] and the saddlepoint method in [7], respectively. As pointed out by Carr and Madan [7], the Fourier inversion method breaks down for deep out-of-the-money options and even generates negative numerical results, e.g. when $K=200$. Moreover, the saddlepoint method may lead to large errors for near the money options, e.g. when $K=100$. In contrast, by choosing $C$ and $N$ using our computable error bounds, the two-sided Laplace inversion algorithm can achieve any desired accuracy, even when the FFT and the saddlepoint method fail.

\subsection{Evaluating the CDF and pricing European call options under the MEM}

In Table 6 we give numerical results for the CDF and European call option prices via the two-sided Laplace inversion algorithm. The upper bounds of both discretization and truncation errors are also reported. In the 'CDF' part, for each $\sigma$, we evaluate $\mathbb{P}\left(X_{t} \leq x_{j}\right)$ for the seven $x_{j}$ given by

$$
x_{j}=\mathbb{E}\left(X_{t}\right)+j \sqrt{\operatorname{var}\left(X_{t}\right)} \text { for } j=0, \pm 1, \pm 2 \text {, and } \pm 3 .
$$

It takes approximately 0.047 seconds to generate all the $14 \mathrm{CDF}$ values and 12 prices. 
TABLE 6: The model parameters are $r=0.05, p_{u}=0.4, q_{d}=0.6, p_{1}=1.2, p_{2}=-0.2, q_{1}=1.3$, $q_{2}=-0.3, \eta_{2}=\theta_{2}=50, S_{0}=K=100, t=1, \lambda=5$, and $\eta_{1}=\theta_{1}=20$ for the 'CDF' part, and $\theta_{1}=\eta_{1}$ for the 'price' part. The algorithm parameters are $\sigma=10, \sigma_{1}^{*}=5, \sigma_{\mathrm{u}}^{*}=15, C=6$, and $N=100$ for the 'CDF' part, and $C=1$ and $N=70$ for the 'price' part. The upper bounds of both the discretization and truncation errors are also reported and are denoted by 'UB of disc. err.' and 'UB of trunc. err.', respectively. The Monte Carlo simulation estimates (denoted by 'MC values') and the associated standard errors (denoted by 'Std. err.') are obtained by simulating 100000 sample paths for the CDF and 1000000 sample paths for prices, with $S_{t}$ used as a control variate to achieve variance reduction. In the 'CDF' part, $x_{j}$ for $j=0, \pm 1, \pm 2$, and \pm 3 are given by (8.2). It takes approximately 0.047 seconds to generate all the $14 \mathrm{CDF}$ values and 12 prices.

\begin{tabular}{|c|c|c|c|c|c|}
\hline \multicolumn{6}{|c|}{ Evaluating the $\mathrm{CDF}$ of the asset return, $\mathbb{P}\left(X_{t} \leq x\right)$, under the MEM } \\
\hline$x$ & Cai, Kou \& Liu & UB of disc. err. & UB of trunc. err. & MC value & Std. err. \\
\hline \multicolumn{6}{|c|}{$\bar{\sigma}=0.2$} \\
\hline$x_{-3}$ & 0.002308272877 & $2.5 \times 10^{-29}$ & $3.9 \times 10^{-22}$ & 0.0024 & 0.000153 \\
\hline$x_{-2}$ & 0.024916216192 & $1.9 \times 10^{-26}$ & $1.4 \times 10^{-22}$ & 0.0254 & 0.000478 \\
\hline$x_{-1}$ & 0.155225832606 & $1.5 \times 10^{-23}$ & $3.4 \times 10^{-23}$ & 0.1553 & 0.000946 \\
\hline$x_{0}$ & 0.496091451231 & $8.2 \times 10^{-21}$ & $7.8 \times 10^{-24}$ & 0.4958 & 0.001008 \\
\hline$x_{1}$ & 0.844951815245 & $3.1 \times 10^{-20}$ & $1.1 \times 10^{-20}$ & 0.8445 & 0.000784 \\
\hline$x_{2}$ & 0.978023723357 & $1.2 \times 10^{-19}$ & $9.1 \times 10^{-18}$ & 0.9775 & 0.000412 \\
\hline$x_{3}$ & 0.998407244203 & $4.4 \times 10^{-19}$ & $4.7 \times 10^{-15}$ & 0.9983 & 0.000125 \\
\hline \multicolumn{6}{|c|}{$\bar{\sigma}=0.3$} \\
\hline$x_{-3}$ & 0.001724821224 & $1.2 \times 10^{-29}$ & $1.4 \times 10^{-42}$ & 0.0017 & 0.000132 \\
\hline$x_{-2}$ & 0.023680606500 & $7.0 \times 10^{-26}$ & $3.1 \times 10^{-45}$ & 0.0240 & 0.000470 \\
\hline$x_{-1}$ & 0.157419494625 & $4.1 \times 10^{-22}$ & $1.4 \times 10^{-48}$ & 0.1582 & 0.000977 \\
\hline$x_{0}$ & 0.498292097431 & $2.4 \times 10^{-18}$ & $8.4 \times 10^{-53}$ & 0.4982 & 0.001032 \\
\hline$x_{1}$ & 0.842575744163 & $1.7 \times 10^{-17}$ & $7.0 \times 10^{-46}$ & 0.8431 & 0.000762 \\
\hline$x_{2}$ & 0.977682915684 & $9.4 \times 10^{-17}$ & $1.8 \times 10^{-39}$ & 0.9775 & 0.000404 \\
\hline$x_{3}$ & 0.998598805036 & $5.3 \times 10^{-16}$ & $9.2 \times 10^{-34}$ & 0.9986 & 0.000113 \\
\hline
\end{tabular}

Pricing European call options under the MEM

\begin{tabular}{ccccccc}
\hline$\eta_{1}$ & $\lambda$ & Cai, Kou \& Liu & UB of disc. err. & UB of trunc. err. & MC value & Std. err. \\
\hline \multicolumn{5}{c}{$\bar{\sigma}=0.2$} \\
\hline 20 & 1 & 10.9747183697 & $1.4 \times 10^{-19}$ & $2.0 \times 10^{-14}$ & 10.970 & 0.00606 \\
& 3 & 11.9448532267 & $5.3 \times 10^{-18}$ & $1.3 \times 10^{-13}$ & 11.938 & 0.00687 \\
& 5 & 12.8307624560 & $2.0 \times 10^{-16}$ & $8.3 \times 10^{-13}$ & 12.827 & 0.00757 \\
\hline 40 & 1 & 10.5757191553 & $2.9 \times 10^{-20}$ & $1.4 \times 10^{-14}$ & 10.572 & 0.00570 \\
& 3 & 10.8205028952 & $4.1 \times 10^{-20}$ & $4.5 \times 10^{-14}$ & 10.816 & 0.00590 \\
& 5 & 11.0584547678 & $5.9 \times 10^{-20}$ & $1.4 \times 10^{-13}$ & 11.053 & 0.00609 \\
\hline & & & $\bar{\sigma}=0.3$ & & \\
\hline 20 & 1 & 14.5975205362 & $5.9 \times 10^{-17}$ & $2.8 \times 10^{-30}$ & 14.594 & 0.00876 \\
& 3 & 15.2999318076 & $2.2 \times 10^{-15}$ & $1.8 \times 10^{-29}$ & 15.293 & 0.00931 \\
& 5 & 15.9667647130 & $8.3 \times 10^{-14}$ & $1.2 \times 10^{-28}$ & 15.964 & 0.00982 \\
\hline 40 & 1 & 14.3163632215 & $1.2 \times 10^{-17}$ & $2.0 \times 10^{-30}$ & 14.315 & 0.00853 \\
& 3 & 14.4847520777 & $1.7 \times 10^{-17}$ & $6.2 \times 10^{-30}$ & 14.482 & 0.00866 \\
& 5 & 14.6507846054 & $2.4 \times 10^{-17}$ & $2.0 \times 10^{-29}$ & 14.647 & 0.00878 \\
\hline
\end{tabular}


TABLE 7: The results in the column 'Cai, Kou \& Liu' are derived via our two-sided Laplace inversion method. The model parameters, the algorithm parameters and $x_{j}$ for $j=0, \pm 1, \pm 2$, and \pm 3 are the same as in Table 6 (the CDF part).

\begin{tabular}{|c|c|c|c|}
\hline \multicolumn{4}{|c|}{ Evaluating the PDF $f_{X_{t}}(x)$ of the MEM process $X_{t}$ at $t=1$} \\
\hline & Cai, Kou \& Liu & UB of disc. err. & UB of trunc. err. \\
\hline & \multicolumn{3}{|c|}{$\bar{\sigma}=0.2$} \\
\hline$f_{X_{1}}\left(x_{-3}\right)$ & 0.022636842044 & $1.2 \times 10^{-27}$ & $1.8 \times 10^{-20}$ \\
\hline$f_{X_{1}}\left(x_{-2}\right)$ & 0.199907611681 & $9.2 \times 10^{-25}$ & $7.0 \times 10^{-21}$ \\
\hline$f_{X_{1}}\left(x_{-1}\right)$ & 0.881184995067 & $7.0 \times 10^{-22}$ & $1.7 \times 10^{-21}$ \\
\hline$f_{X_{1}}\left(x_{0}\right)$ & 1.535829395291 & $4.0 \times 10^{-19}$ & $4.1 \times 10^{-22}$ \\
\hline$f_{X_{1}}\left(x_{1}\right)$ & 0.915712684352 & $1.5 \times 10^{-18}$ & $5.6 \times 10^{-19}$ \\
\hline$f_{X_{1}}\left(x_{2}\right)$ & 0.192067651206 & $5.7 \times 10^{-18}$ & $4.4 \times 10^{-16}$ \\
\hline$f_{X_{1}}\left(x_{3}\right)$ & 0.017364051469 & $2.1 \times 10^{-17}$ & $2.2 \times 10^{-13}$ \\
\hline
\end{tabular}

In Table 7 we report the numerical results for the PDF $f_{X_{t}}(x)$ of the MEM process $X_{t}$ at $t=1$ via our two-sided Laplace inversion algorithm. Applying the results obtained in Sections 6.2-6.4, we also provide the associated upper bounds of discretization and truncation errors. Owing to the space restriction, we omit the numerical results in the case of $\bar{\sigma}=0.3$ which are available upon request.

\subsection{Pricing lookback options under the DEM}

In Table 8 we provide numerical prices for the lookback put options under the DEM as well as associated discretization and truncation error bounds obtained via our Laplace inversion algorithm. It can be seen that our algorithm is quite efficient because it takes only 0.01 seconds to produce one numerical price via our method.

TABLE 8: The model parameters are $r=0.05, \bar{\sigma}=0.4, \lambda=3, p=0.6, \eta=20, \theta=15, S_{0}=10$, and $T=1$. The algorithm parameters are $\sigma=2, C=6$, and $N=5000$. The upper bounds of both the discretization and truncation errors are also reported and are denoted by 'UB of disc. err.' and 'UB of trunc. err.', respectively. The Monte Carlo simulation estimates (denoted by 'MC values') and the associated standard errors (denoted by 'Std. err.') are obtained by simulating 50000 sample paths and setting the step size to be 0.00002 . It takes approximately 0.01 seconds to generate one numerical price via our algorithm.

\begin{tabular}{cccccc}
\hline \multicolumn{5}{c}{ Pricing lookback put options under the DEM } \\
\hline$M$ & Cai, Kou \& Liu & UB of disc. err. & UB of trunc. err. & MC values & Std. err. \\
\hline 11.0 & 3.58305 & $5.5 \times 10^{-7}$ & $5.8 \times 10^{-4}$ & 3.581 & 0.00807 \\
11.5 & 3.69932 & $5.6 \times 10^{-7}$ & $6.1 \times 10^{-4}$ & 3.697 & 0.00799 \\
12.0 & 3.85479 & $5.7 \times 10^{-7}$ & $6.3 \times 10^{-4}$ & 3.852 & 0.00791 \\
12.5 & 4.04576 & $5.8 \times 10^{-7}$ & $6.6 \times 10^{-4}$ & 4.043 & 0.00783 \\
13.0 & 4.26878 & $5.9 \times 10^{-7}$ & $6.9 \times 10^{-4}$ & 4.266 & 0.00776 \\
13.5 & 4.52060 & $6.0 \times 10^{-7}$ & $7.1 \times 10^{-4}$ & 4.518 & 0.00768 \\
14.0 & 4.79822 & $6.1 \times 10^{-7}$ & $7.4 \times 10^{-4}$ & 4.797 & 0.00759 \\
14.5 & 5.09887 & $6.2 \times 10^{-7}$ & $7.6 \times 10^{-4}$ & 5.098 & 0.00749 \\
15.0 & 5.42002 & $6.3 \times 10^{-7}$ & $7.9 \times 10^{-4}$ & 5.419 & 0.00738 \\
\hline
\end{tabular}




\section{Appendix A. Proofs of Theorem 3.1 and Lemma 3.1}

Proof of Theorem 3.1. To prove (3.1), it suffices to show that, for any $t \neq 0$ and $\sigma \in$ ROAC,

$$
f(t)=\frac{\mathrm{e}^{\sigma t} L_{f}(\sigma)}{2|t|}+\frac{\mathrm{e}^{\sigma t}}{|t|} \sum_{k=1}^{\infty}(-1)^{k} \operatorname{Re}\left(L_{f}\left(\sigma+\frac{k \pi \mathrm{i}}{t}\right)\right)-\sum_{k=-\infty, k \neq 0}^{+\infty} \mathrm{e}^{-2 \sigma k t} f((2 k+1) t)
$$

with the last term representing the discretization error. Indeed, to evaluate $f(t)$, we can alternatively apply (A.1) to the function $g_{+}(y):=f(y-C)$ at the point $t+C$ if $t \geq 0$ or to the function $g_{-}(y):=f(y+C)$ at the point $t-C$ if $t<0$. This yields (3.1) immediately.

Now we start to prove (A.1). Because $\sigma \in \operatorname{ROAC}$, substituting $\sigma+\mathrm{i} \omega$ for $s$ in the Bromwich contour integral

$$
f(t)=\frac{1}{2 \pi \mathrm{i}} \lim _{T \rightarrow+\infty} \int_{\sigma-\mathrm{i} T}^{\sigma+\mathrm{i} T} \mathrm{e}^{t s} L_{f}(s) \mathrm{d} s
$$

we obtain

$$
\begin{aligned}
f(t) & =\frac{\mathrm{e}^{\sigma t}}{2 \pi} \lim _{T \rightarrow+\infty} \int_{\sigma-\mathrm{i} T}^{\sigma+\mathrm{i} T}[\cos (\omega t)+\mathrm{i} \sin (\omega t)] L_{f}(\sigma+\mathrm{i} \omega) \mathrm{d} \omega \\
= & \frac{\mathrm{e}^{\sigma t}}{2 \pi} \int_{0}^{+\infty}\left\{[\cos (\omega t)+\mathrm{i} \sin (\omega t)] L_{f}(\sigma+\mathrm{i} \omega)\right. \\
& \left.+[\cos (\omega t)-\mathrm{i} \sin (\omega t)] L_{f}(\sigma-\mathrm{i} \omega)\right\} \mathrm{d} \omega . \\
= & \frac{\mathrm{e}^{\sigma t}}{\pi} \int_{0}^{+\infty}\left[\cos (\omega t) \operatorname{Re}\left(L_{f}(\sigma+\mathrm{i} \omega)\right)+\sin (\omega t) \operatorname{Im}\left(L_{f}(\sigma-\mathrm{i} \omega)\right)\right] \mathrm{d} \omega,
\end{aligned}
$$

where the last equality holds because $\operatorname{Re}\left(L_{f}(\sigma+\mathrm{i} \omega)\right)=\operatorname{Re}\left(L_{f}(\sigma-\mathrm{i} \omega)\right)$ and $\operatorname{Im}\left(L_{f}(\sigma+\mathrm{i} \omega)\right)=$ $-\operatorname{Im}\left(L_{f}(\sigma-\mathrm{i} \omega)\right)$. By the trapezoidal rule, for $h>0$, we define $\tilde{f}(t)$ as

$$
\begin{aligned}
\tilde{f}(t):= & \frac{h \mathrm{e}^{\sigma t}}{2 \pi} L_{f}(\sigma)+\frac{h \mathrm{e}^{\sigma t}}{\pi} \sum_{k=1}^{+\infty} \cos (k h t) \operatorname{Re}\left(L_{f}(\sigma+\mathrm{i} k h)\right) \\
& +\frac{h \mathrm{e}^{\sigma t}}{\pi} \sum_{k=1}^{+\infty} \sin (k h t) \operatorname{Im}\left(L_{f}(\sigma-\mathrm{i} k h)\right) .
\end{aligned}
$$

Because $t \neq 0$, letting $h=\pi /|t|$ yields

$$
\tilde{f}(t)=\frac{\mathrm{e}^{\sigma t} L_{f}(\sigma)}{2|t|}+\frac{\mathrm{e}^{\sigma t}}{|t|} \sum_{k=1}^{\infty}(-1)^{k} \operatorname{Re}\left(L_{f}\left(\sigma+\frac{k \pi \mathrm{i}}{t}\right)\right) .
$$

To analyze the discretization error $\tilde{f}(t)-f(t)$ generated by the trapezoidal rule, we define $g(x):=\mathrm{e}^{-\sigma x} f(x)$ for any fixed $\sigma \in$ ROAC. Under the condition of Theorem 3.1, $g(x)$ is absolutely integrable over $\mathbb{R}$, is of bounded variation on $\mathbb{R}$, and satisfies $2 g(x)=g(x+0)+$ $g(x-0)$ for any $x$. Then, for any fixed $t \neq 0, g^{*}(x):=g(t+x / h)=g(t+|t| x / \pi)$ also has these three properties. Applying the Poisson summation formula to $g^{*}(x)$ yields (see, e.g. Equation (13.4) of [15, p. 68])

$$
\sum_{k=-\infty}^{+\infty} g^{*}(2 \pi k)=\frac{1}{2 \pi} \sum_{k=-\infty}^{+\infty} \int_{-\infty}^{+\infty} g^{*}(z) \mathrm{e}^{-\mathrm{i} k z} \mathrm{~d} z
$$


The left-hand side of (A.3) is equal to

$$
\begin{aligned}
\sum_{k=-\infty}^{+\infty} g(t+2|t| k) & =\sum_{k=-\infty}^{+\infty} g((2 k+1) t) \\
& =\sum_{k=-\infty}^{+\infty} \mathrm{e}^{-\sigma(2 k+1) t} f((2 k+1) t) \\
& =\mathrm{e}^{-\sigma t}\left[f(t)+\sum_{k=-\infty, k \neq 0}^{+\infty} \mathrm{e}^{-2 \sigma k t} f((2 k+1) t)\right]
\end{aligned}
$$

whereas the right-hand side of (A.3) is given by

$$
\begin{aligned}
& \frac{1}{2 \pi} \sum_{k=-\infty}^{+\infty} \int_{-\infty}^{+\infty} g\left(t+\frac{|t| z}{\pi}\right) \mathrm{e}^{-\mathrm{i} k z} \mathrm{~d} z \\
& \quad=\frac{1}{2|t|} \sum_{k=-\infty}^{+\infty}\left[\left(\int_{-\infty}^{+\infty} g(y) \mathrm{e}^{-\mathrm{i} k \pi y /|t|} \mathrm{d} y\right) \mathrm{e}^{\mathrm{i} k \pi t /|t|}\right] \\
& \quad=\frac{1}{2|t|} \sum_{k=-\infty}^{+\infty} L_{f}\left(\sigma+\frac{\mathrm{i} k \pi}{|t|}\right) \mathrm{e}^{\mathrm{i} k \pi t /|t|} \\
& \quad=\frac{1}{2|t|} \sum_{k=-\infty}^{+\infty}(-1)^{k} L_{f}\left(\sigma+\frac{\mathrm{i} k \pi}{t}\right) \\
& =\frac{1}{2|t|} L_{f}(\sigma)+\frac{1}{|t|} \sum_{k=1}^{+\infty}(-1)^{k} \operatorname{Re}\left(L_{f}\left(\sigma+\frac{\mathrm{i} k \pi}{t}\right)\right),
\end{aligned}
$$

where the first equality holds because of the change of variable $y=t+|t| z / \pi$. Equating (A.4) and (A.5) yields (A.1) immediately. The proof is completed by comparing (A.1) with (A.2).

Proof of Lemma 3.1. It suffices to show that, for any $\sigma \in$ ROAC, $\mathrm{e}^{-\sigma x} f(x)$ satisfies (i) $\mathrm{e}^{-\sigma x} f(x) \in \mathcal{C}^{1}$; (ii) $\int_{-\infty}^{+\infty} \mathrm{e}^{-\sigma x}|f(x)| \mathrm{d} x<+\infty$; and (iii) $\int_{-\infty}^{+\infty}\left|\left(\mathrm{e}^{-\sigma x} f(x)\right)^{\prime}\right| \mathrm{d} x<+\infty$. Conditions (i) and (ii) hold obviously. As regards (iii), since $f(x)=\mathrm{e}^{c x} \bar{f}(x)$ with $\bar{f}(x)$ a monotone function, without loss of generality, we assume that $\bar{f}(x)$ is nondecreasing, i.e. $\bar{f}^{\prime}(x) \geq 0$. Then

$$
\begin{aligned}
\int_{-\infty}^{+\infty}\left|\left(\mathrm{e}^{-\sigma x} f(x)\right)^{\prime}\right| \mathrm{d} x & =\int_{-\infty}^{+\infty}\left|(c-\sigma) \mathrm{e}^{(c-\sigma) x} \bar{f}(x)+\mathrm{e}^{(c-\sigma) x} \bar{f}^{\prime}(x)\right| \mathrm{d} x \\
& \leq|c-\sigma| \int_{-\infty}^{+\infty}\left|\mathrm{e}^{(c-\sigma) x} \bar{f}(x)\right| \mathrm{d} x+\int_{-\infty}^{+\infty} \mathrm{e}^{(c-\sigma) x} \bar{f}^{\prime}(x) \mathrm{d} x \\
& =|c-\sigma| \int_{-\infty}^{+\infty}\left|\mathrm{e}^{-\sigma x} f(x)\right| \mathrm{d} x-(c-\sigma) \int_{-\infty}^{+\infty} \mathrm{e}^{-\sigma x} f(x) \mathrm{d} x \\
& <+\infty
\end{aligned}
$$

where the last equality holds because of integration by parts. 


\section{Appendix B. Proof of Theorem 4.1}

From (4.1) we obtain

$$
|f(y)| \leq \begin{cases}\delta\left(\sigma_{1}^{*}\right) \mathrm{e}^{\sigma_{1}^{*} y} & \text { for any } y \geq 0 \\ \delta\left(\sigma_{\mathrm{u}}^{*}\right) \mathrm{e}^{\sigma_{\mathrm{u}}^{*} y} & \text { for any } y \leq 0\end{cases}
$$

We proceed to discuss two cases, $t \geq 0$ and $t<0$. If $t \geq 0$ then, for any $C \geq 0$, we have

$$
2 k(t+\operatorname{sgn}(t) C)+t=2 k(t+C)+t \begin{cases}\geq 0 & \text { if } k>0 \text { and } t \geq 0 \\ \leq 0 & \text { if } k<0 \text { and } t \geq 0\end{cases}
$$

Then by (B.1) we obtain, for any fixed $t \geq 0, \sigma \in\left(\sigma_{1}^{*}, \sigma_{\mathrm{u}}^{*}\right)$, and $C>0$,

$$
\begin{aligned}
\left|e_{\mathrm{D}}(t, \sigma, C)\right| & =\left|\sum_{k=-\infty, k \neq 0}^{\infty} \mathrm{e}^{-2 k(t+C) \sigma} f(2 k(t+C)+t)\right| \\
& \leq \sum_{k=-\infty, k \neq 0}^{\infty} \mathrm{e}^{-2 k(t+C) \sigma}|f(2 k(t+C)+t)| \\
& \leq \delta\left(\sigma_{\mathrm{u}}^{*}\right) \sum_{k=-\infty}^{-1} \mathrm{e}^{-2 k(t+C) \sigma} \mathrm{e}^{\sigma_{\mathrm{u}}^{*}[2 k(t+C)+t]}+\delta\left(\sigma_{1}^{*}\right) \sum_{k=1}^{\infty} \mathrm{e}^{-2 k(t+C) \sigma} \mathrm{e}^{\sigma_{1}^{*}[2 k(t+C)+t]} \\
& =\delta\left(\sigma_{\mathrm{u}}^{*}\right) \mathrm{e}^{\left(2 \sigma-\sigma_{\mathrm{u}}^{*}\right) t} \frac{\mathrm{e}^{-2 C\left(\sigma_{\mathrm{u}}^{*}-\sigma\right)}}{1-\mathrm{e}^{-2(t+C)\left(\sigma_{\mathrm{u}}^{*}-\sigma\right)}+\delta\left(\sigma_{1}^{*}\right) \mathrm{e}^{\left(3 \sigma_{1}^{*}-2 \sigma\right) t} \frac{\mathrm{e}^{-2 C\left(\sigma-\sigma_{1}^{*}\right)}}{1-\mathrm{e}^{-2(t+C)\left(\sigma-\sigma_{1}^{*}\right)}}} \\
& \leq \delta\left(\sigma_{\mathrm{u}}^{*}\right) \mathrm{e}^{\left(2 \sigma-\sigma_{\mathrm{u}}^{*}\right) t} \frac{\mathrm{e}^{-2 C\left(\sigma_{\mathrm{u}}^{*}-\sigma\right)}}{1-\mathrm{e}^{-2 C\left(\sigma_{\mathrm{u}}^{*}-\sigma\right)}}+\delta\left(\sigma_{1}^{*}\right) \mathrm{e}^{\left(3 \sigma_{1}^{*}-2 \sigma\right) t} \frac{\mathrm{e}^{-2 C\left(\sigma-\sigma_{1}^{*}\right)}}{1-\mathrm{e}^{-2 C\left(\sigma-\sigma_{1}^{*}\right)}} \\
& =\delta\left(\sigma_{\mathrm{u}}^{*}\right) \mathrm{e}^{\left(2 \sigma-\sigma_{\mathrm{u}}^{*}\right) t} \frac{1}{\mathrm{e}^{2 C\left(\sigma_{\mathrm{u}}^{*}-\sigma\right)}-1}+\delta\left(\sigma_{1}^{*}\right) \mathrm{e}^{\left(3 \sigma_{1}^{*}-2 \sigma\right) t} \frac{1}{\mathrm{e}^{2 C\left(\sigma-\sigma_{1}^{*}\right)}-1} \\
& \leq \frac{\rho(\sigma, t)}{\mathrm{e}^{\theta(\sigma) C}-1}, \quad
\end{aligned}
$$

which is exactly (4.2).

If $t<0$, we have, for any $C \geq 0$,

$$
2 k(t+\operatorname{sgn}(t) C)+t=2 k(t-C)+t \begin{cases}\geq 0 & \text { if } k<0 \text { and } t<0, \\ \leq 0 & \text { if } k>0 \text { and } t<0 .\end{cases}
$$

By (B.1), the discretization error for any $t<0, \sigma \in\left(\sigma_{1}^{*}, \sigma_{\mathrm{u}}^{*}\right)$, and $C>0$ can be bounded as follows:

$$
\begin{aligned}
\left|e_{\mathrm{D}}(t, \sigma, C)\right| & =\left|\sum_{k=-\infty, k \neq 0}^{\infty} \mathrm{e}^{-2 k(t-C) \sigma} f(2 k(t-C)+t)\right| \\
& \leq \sum_{k=-\infty, k \neq 0}^{\infty} \mathrm{e}^{-2 k(t-C) \sigma}|f(2 k(t-C)+t)| \\
& \leq \delta\left(\sigma_{\mathrm{u}}^{*}\right) \sum_{k=1}^{\infty} \mathrm{e}^{-2 k(t-C) \sigma} \mathrm{e}^{\sigma[2 k(t-C)+t]}+\delta\left(\sigma_{1}^{*}\right) \sum_{k=-\infty}^{-1} \mathrm{e}^{-2 k(t-C) \sigma} \mathrm{e}^{\sigma_{1}^{*}[2 k(t-C)+t]}
\end{aligned}
$$




$$
\begin{aligned}
& =\delta\left(\sigma_{\mathrm{u}}^{*}\right) \mathrm{e}^{\left(3 \sigma_{\mathrm{u}}^{*}-2 \sigma\right) t} \frac{\mathrm{e}^{-2 C\left(\sigma_{\mathrm{u}}^{*}-\sigma\right)}}{1-\mathrm{e}^{-2(C-t)\left(\sigma_{\mathrm{u}}^{*}-\sigma\right)}}+\delta\left(\sigma_{1}^{*}\right) \mathrm{e}^{\left(2 \sigma-\sigma_{1}^{*}\right) t} \frac{\mathrm{e}^{-2 C\left(\sigma-\sigma_{1}^{*}\right)}}{1-\mathrm{e}^{-2(C-t)\left(\sigma-\sigma_{1}^{*}\right)}} \\
& \leq \delta\left(\sigma_{\mathrm{u}}^{*}\right) \mathrm{e}^{\left(3 \sigma_{\mathrm{u}}^{*}-2 \sigma\right) t} \frac{\mathrm{e}^{-2 C\left(\sigma_{\mathrm{u}}^{*}-\sigma\right)}}{1-\mathrm{e}^{-2 C\left(\sigma_{\mathrm{u}}^{*}-\sigma\right)}}+\delta\left(\sigma_{1}^{*}\right) \mathrm{e}^{\left(2 \sigma-\sigma_{1}^{*}\right) t} \frac{\mathrm{e}^{-2 C\left(\sigma-\sigma_{1}^{*}\right)}}{1-\mathrm{e}^{-2 C\left(\sigma-\sigma_{1}^{*}\right)}} \\
& =\delta\left(\sigma_{\mathrm{u}}^{*}\right) \mathrm{e}^{\left(3 \sigma_{\mathrm{u}}^{*}-2 \sigma\right) t} \frac{1}{\mathrm{e}^{2 C\left(\sigma_{\mathrm{u}}^{*}-\sigma\right)}-1}+\delta\left(\sigma_{1}^{*}\right) \mathrm{e}^{\left(2 \sigma-\sigma_{\mathrm{l}}^{*}\right) t} \frac{1}{\mathrm{e}^{2 C\left(\sigma-\sigma_{1}^{*}\right)}-1} \\
& \leq \frac{\rho(\sigma, t)}{\mathrm{e}^{\theta(\sigma) C}-1} .
\end{aligned}
$$

This completes the proof.

\section{Appendix C. Proof of Theorem 5.1}

By (3.2) we obtain

$$
\begin{aligned}
\left|e_{\mathrm{T}}(t, \sigma, C, N)\right| & \leq \frac{\mathrm{e}^{\sigma t}}{|t|+C} \sum_{k=N+1}^{+\infty}\left|\operatorname{Re}\left(\exp \left(-\frac{\operatorname{sgn}(t) C k \pi \mathrm{i}}{t+\operatorname{sgn}(t) C}\right) L_{f}\left(\sigma+\frac{k \pi \mathrm{i}}{t+\operatorname{sgn}(t) C}\right)\right)\right| \\
& \leq \frac{\mathrm{e}^{\sigma t}}{|t|+C} \sum_{k=N+1}^{+\infty}\left|L_{f}\left(\sigma+\frac{k \pi \mathrm{i}}{t+\operatorname{sgn}(t) C}\right)\right| .
\end{aligned}
$$

If $L_{f}(\sigma+\mathrm{i} \omega)$ satisfies (5.1) then (5.2) follows because, for any $N>(|t|+C) \omega^{*} / \pi-1$,

$$
\begin{aligned}
\left|e_{\mathrm{T}}(t, \sigma, C, N)\right| & \leq \frac{\mathrm{e}^{\sigma t}}{|t|+C} \sum_{k=N+1}^{+\infty} \zeta(\sigma)\left(\frac{k \pi}{|t|+C}\right)^{-(1+\rho)} \\
& =\frac{\zeta(\sigma) \mathrm{e}^{\sigma t}(|t|+C)^{\rho}}{\pi^{1+\rho}} \sum_{k=N+1}^{+\infty} k^{-(1+\rho)} \\
& \leq \frac{\zeta(\sigma) \mathrm{e}^{\sigma t}(|t|+C)^{\rho}}{\pi^{1+\rho}} \int_{N}^{+\infty} y^{-(1+\rho)} \mathrm{d} y \\
& =\frac{\zeta(\sigma) \mathrm{e}^{\sigma t}(|t|+C)^{\rho}}{\rho \pi^{1+\rho}} N^{-\rho}
\end{aligned}
$$

where the last inequality holds due to the monotonicity of the function $y^{-(1+\rho)}$ on $[N,+\infty)$.

Similarly, if $L_{f}(\sigma+\mathrm{i} \omega)$ satisfies (5.3), we have for any $N>(|t|+C) \omega^{*} / \pi-1$,

$$
\begin{aligned}
\left|e_{\mathrm{T}}(t, \sigma, C, N)\right| & \leq \frac{\zeta(\sigma) \mathrm{e}^{\sigma t} \alpha^{-\beta / \xi}}{|t|+C} \sum_{k=N+1}^{+\infty} k^{-\beta} \mathrm{e}^{-\rho \alpha k^{\xi}} \\
& \leq \frac{\zeta(\sigma) \mathrm{e}^{\sigma t} \alpha^{-\beta / \xi}}{|t|+C} \int_{N}^{+\infty} y^{-\beta} \mathrm{e}^{-\rho \alpha y^{\xi}} \mathrm{d} y \\
& =\frac{\zeta(\sigma) \mathrm{e}^{\sigma t}}{\pi \xi \rho^{(1-\beta) / \xi}} \Gamma\left(\frac{1-\beta}{\xi}, \rho \alpha N^{\xi}\right) .
\end{aligned}
$$

By the following result about the asymptotic behavior of the upper incomplete gamma function (see, e.g. [13, p. 66])

$$
\lim _{x \rightarrow+\infty} \frac{\Gamma(s, x)}{x^{s-1} \mathrm{e}^{-x}}=1,
$$

we obtain (5.4) immediately. 


\section{Acknowledgements}

We thank the Editor, the Associate Editor, and an anonymous referee for their constructive suggestions that significantly helped to improve our paper. We are very grateful to Paul Glasserman, Liming Feng, Chao Shi, and Ward Whitt for their helpful comments. The first author is also grateful for financial support from the GRF of the Hong Kong RGC (project number 610711) and the Direct Allocation Grant (project number DAG11EG07G). The second author was supported by a start-up grant for research on quantitative finance at National University of Singapore.

\section{References}

[1] Aвate, J. And Whitt, W. (1992). The Fourier-series method for inverting transforms of probability distributions. Queueing Systems 10, 5-87.

[2] Broadie, M. and Yamamoto, Y. (2003). Application of the fast Gauss transform to option pricing. Manag. Sci. 49, 1071-1088.

[3] CaI, N. and Kou, S. G. (2011). Option pricing under a mixed-exponential jump diffusion model. Manag. Sci. 57, 2067-2081.

[4] CaI, N., Kou, S. G. ANd LiU, Z. (2013). A two-sided Laplace inversion algorithm with computable error bounds and its application in financial engineering. Electronic companion. Available at http://ihome.ust.hk/ $\sim$ ningcai/index_IELM_1.html.

[5] CaI, N., Peng, X. AND SHI, C. (2013). Maximum-likelihood estimation via two-sided Laplace inversion with error control. Working paper.

[6] Carr, P. And Madan, D. B. (1999). Option valuation using the fast Fourier transform. J. Computational Finance 2, 61-73.

[7] Carr, P. and Madan, D. (2009). Saddlepoint methods for option pricing. J. Computational Finance 13, 49-61.

[8] Feng, L. AND Lin, X. (2013). Inverting analytic characteristic functions and financial applications. SIAM J. Financial Math. 4, 372-398.

[9] Feng, L. and Linetsky, V. (2008). Pricing discretely monitored barrier options and defaultable bonds in Lévy process models: a fast Hilbert transform approach. Math. Finance 18, 337-384.

[10] Feng, L. AND Linetsky, V. (2009). Computing exponential moments of the discrete maximum of a Lévy process and lookback options. Finance Stoch. 13, 501-529.

[11] Kou, S. G. AND WANG, H. (2004). Option pricing under a double exponential jump diffusion model. Manag. Sci. 50, 1178-1192.

[12] LEE, R. W. (2004). Option pricing by transform methods: extensions, unification, and error control. J. Computational Finance 7, 51-86.

[13] Olver, F. W. J. (1974). Asymptotics and Special Functions. Academic Press, New York.

[14] Petrella, G. (2004). An extension of the Euler Laplace transform inversion algorithm with applications in option pricing. Operat. Res. Lett. 32, 380-389.

[15] Zygmund, A. (1968). Trigonometric Series, 2nd edn. Cambridge University Press. 